\title{
On Cartesian stiffness matrices in rigid body dynamics: an energetic perspective
}

\author{
Melodie F. Metzger • Nur Adila Faruk Senan • \\ Oliver M. O'Reilly
}

Received: 17 April 2009 / Accepted: 8 April 2010 / Published online: 12 May 2010

(C) The Author(s) 2010. This article is published with open access at Springerlink.com

\begin{abstract}
Several Cartesian stiffness matrices for a single rigid body subject to a conservative force field are developed in this paper. The treatment is based on energetic arguments and an Euler angle parameterization of the rotation of the rigid body is employed. Several new representations for the stiffness matrix are obtained and the relation to other works on Cartesian stiffness matrices and Hessians is illuminated. Additional details are presented with respect to determining the Cartesian stiffness matrix for a pair of rigid bodies, as well as for a system of rigid bodies constrained to a plane.
\end{abstract}

Keywords Rigid body $\cdot$ Rotation $\cdot$ Stiffness matrix $\cdot$ Cartesian stiffness matrix $\cdot$ Dual Euler basis $\cdot$ Euler angles $\cdot$ Conservative force fields

\section{Introduction}

Papers by Duffy, Griffis, and Pigoski [8, 18] appeared in the early 1990s discussing examples of a linear mapping of the increments to the conservative force and moment components acting on a rigid body with the infinitesimal displacements and rotations which produced them. The linear mapping was a stiffness matrix $\mathrm{K}_{O}$ which had the unusual feature of being asymmetric. To distinguish this matrix from the Hessian $\mathrm{H}$ of a potential energy, $\mathrm{K}_{O}$ is known as the "Cartesian stiffness matrix." Griffis and Duffy's examples in [8] featured rigid bodies tethered to a fixed surface using linear springs. In the event that the springs were unstretched

\footnotetext{
M.F. Metzger

Department of Orthopaedic Surgery, University of California at San Francisco, San Francisco, CA 94110, USA

e-mail: metzgerm@orthosurg.ucsf.edu
}

N.A. Faruk Senan · O.M. O’Reilly (凶)

Department of Mechanical Engineering, University of California at Berkeley, Berkeley,

CA 94706-1740, USA

e-mail: oreilly@berkeley.edu

N.A. Faruk Senan

e-mail: adilafaruk@berkeley.edu 
in the state of the body of interest, then the asymmetry of $\mathrm{K}_{O}$ was seen to vanish. Subsequent works by Ciblak and Lipkins [5] clarified aspects of the asymmetry. Howard et al. [9] and Žefran and Kumar [21] used a Lie group approach, considered the more general case of a rigid body in a potential field, and established several representations for the Cartesian stiffness matrix. Several others researchers, such as [3, 10, 19], extended the formulation of the Cartesian stiffness matrices to a range of mechanical systems. Our interest in this matrix stems from its potential biomechanical applications ranging from quantifying the motion of a knee joint using a stiffness parameter [1], to modeling the intervertebral disc of the spine using a stiffness matrix $[7,16,17]$. These joints are ideally suited to such an analysis especially in the realm of small motions about an equilibrium.

Here, we present another perspective on the Cartesian stiffness matrix, and discuss its dependency on the force-moment pair that it characterizes. In contrast to the works [5, $8-10,18,21]$, we do not use screw theory to describe the rigid body motion, and instead parameterize the rotation of the rigid body using a set of Euler angles. We follow [15] and use an argument based on energetic considerations to establish the resultant conservative force and moment acting on a rigid body. Invoking the same argument, expressions for the conservative moment considered relative to a fixed point $O$, an arbitrary material point $A$, and the center of mass $\bar{X}$ are established. These expressions feature equivalent (but distinct) functional forms of the potential energy function. With the help of a Taylor series expansion, several examples of Cartesian stiffness matrices are then established: ${ }^{1}$

$$
\begin{aligned}
& { }_{1} \mathrm{~K}_{c}=\left[\begin{array}{cc}
\mathrm{Q}^{T} & 0 \\
0 & \mathrm{G}^{T}
\end{array}\right]{ }_{1} \mathrm{H}\left[\begin{array}{cc}
\mathrm{Q} & 0 \\
0 & \mathrm{G}
\end{array}\right]+\left[\begin{array}{ll}
0 & { }_{1} \mathrm{C} \\
0 & { }_{1} \mathrm{D}
\end{array}\right]+\left[\begin{array}{ll}
0 & { }_{1} \mathrm{Y} \\
0 & { }_{1} \mathrm{Z}
\end{array}\right], \\
& { }_{2} \mathrm{~K}_{c}=\left[\begin{array}{cc}
\mathrm{I} & 0 \\
0 & \mathrm{G}^{T}
\end{array}\right]{ }_{2} \mathrm{H}\left[\begin{array}{ll}
\mathrm{I} & 0 \\
0 & \mathrm{G}
\end{array}\right]+\left[\begin{array}{cc}
0 & 0 \\
0 & { }_{2} \mathrm{D}
\end{array}\right] .
\end{aligned}
$$

The conditions required for the asymmetry of these matrices are discussed at length. The stiffness matrix ${ }_{1} \mathrm{~K}_{c}$ is related to the stiffness matrix $\mathrm{K}_{O}$ analyzed in Ciblak and Lipkins [5], Howard et al. [9], and Žefran and Kumar [21]:

$$
{ }_{1} \mathrm{~K}_{c}=\mathrm{K}_{O}+\left[\begin{array}{ll}
0 & { }_{1} \mathrm{Y} \\
0 & { }_{1} \mathrm{Z}
\end{array}\right] .
$$

We also discuss the steps necessary to extrapolate the results obtained to systems composed of more than a single rigid body and elaborate further on this by examining planar motions of multiple rigid bodies. We illuminate further features of the stiffness matrices using a mass-spring system known as the Stewart-Gough platform that is featured in Griffis and Duffy [8], among others. Finally, we contrast the Cartesian stiffness matrix and the stiffness matrix formed from the Hessian $\mathrm{H}$.

An outline of the paper is as follows. In the next section, relevant background from a variety of sources on the kinematics and kinetics of a rigid body is presented. Using an argument based on energetics, several representations for the conservative forces and moments acting on the rigid body are established in Sect. 3. These representations are used to establish expressions for various Cartesian stiffness matrices in Sect. 4. The skew-symmetric parts of these stiffness matrices receive additional attention in Sect. 5. There it is shown how the skew-symmetric parts are related to the conservative forces and moments needed for

\footnotetext{
${ }^{1}$ These representations are established in Sect. 4 (cf. (28) and (35) in particular).
} 
equilibrium. Several of the results in this section can be considered as analogues of those presented in $[5,9,21]$. The expressions for the stiffness matrices are then applied to a class of planar mechanisms in Sect. 6. Following $[8,9,21]$ and others, the Stewart-Gough platform and its associated values of ${ }_{1} \mathrm{~K}_{c}$ and ${ }_{2} \mathrm{~K}_{c}$ are discussed in Sect. 7.

Section 8 further expands upon the analysis of Sects. 3 and 4 by examining systems composed of more than one rigid body. A planar multibody system is analyzed in Sect. 9 to further illuminate the results obtained in Sect. 8. Some closing remarks are presented in Sect. 10 and the two stiffness matrices characterized by the Cartesian stiffness matrix $\mathrm{K}_{c}$ and the Hessian $\mathrm{H}$ will be compared there.

The paper contains two appendices. The first appendix, Appendix A, presents proofs of certain identities which are needed to establish several results in Sect. 5. Details on the 3-2-1 set of Euler angles which are used in Sect. 7 are presented in Appendix B.

\subsection{Notation}

In the present paper, arrays of real numbers are denoted by san-serif roman letters, such as $\mathbf{G}, \mathbf{x}$, etc. Vectors and tensors are denoted by bold-faced roman letters, e.g., $\mathbf{x}$ and $\mathbf{C}$. The indices $i, j, k, l, n, m, r$, and $s$ range from 1 to 3 . Further details on notation can be found in Appendix A.

\section{Background}

A rigid body $\mathcal{B}$ consists of a collection of material points $X$ where the distance between any of these points remains constant. As shown in Fig. 1, it is convenient to define a fixed reference configuration $\kappa_{0}$ of this body. This configuration occupies a fixed region of Euclidean three-space $\mathbb{E}^{3}$. The position vector, relative to a fixed origin $O$, of a material point $X$ in this configuration is defined by the position vector $\mathbf{X}$. In a similar manner, the present (or current) configuration $\kappa_{t}$ of $\mathcal{B}$ can be defined and the position vector of a material point $X$ in this configuration is denoted by $\mathbf{x}$.

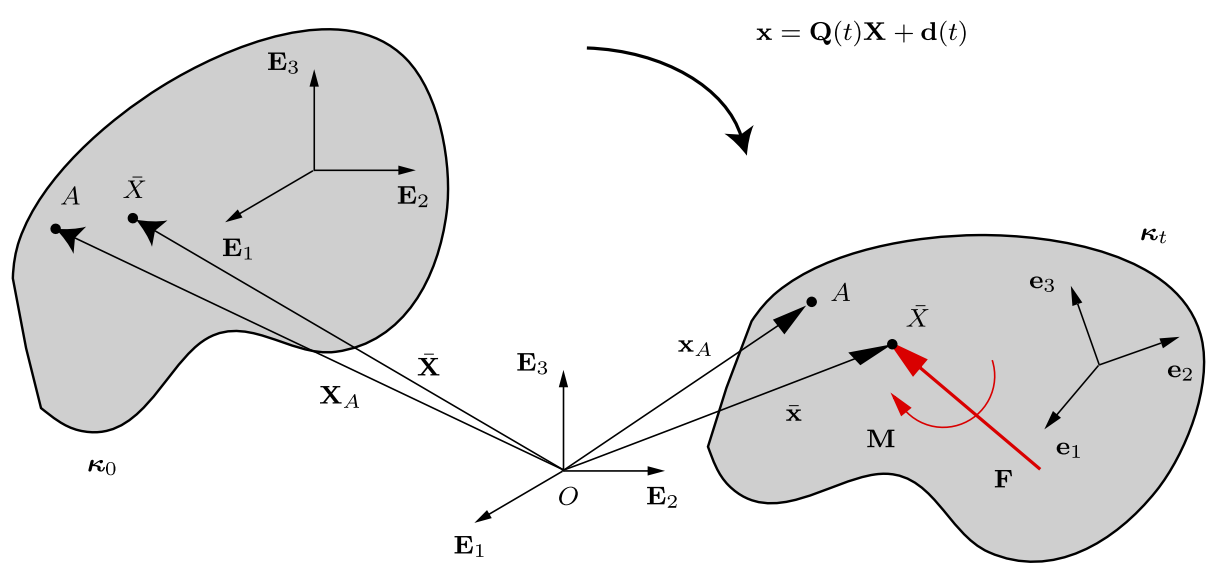

Fig. 1 The reference $\kappa_{0}$ and present $\kappa_{t}$ configurations of a rigid body $\mathcal{B}$. This figure also displays the corotational basis $\left\{\mathbf{e}_{1}, \mathbf{e}_{2}, \mathbf{e}_{3}\right\}$, center of mass $\bar{X}$, material point $A$, and the resultant force $\mathbf{F}$ and moment $\mathbf{M}$ 
The motion of the rigid body can be characterized by the rotation $\mathbf{Q}$ of the body and the position vector $\mathbf{x}$ of a point on the body:

$$
\mathbf{x}=\mathbf{Q}(t) \mathbf{X}+\mathbf{d}(t) .
$$

Here, $\mathbf{d}(t)$ is a vector-valued function of time, and $\mathbf{Q}(t)$ is a rotation tensor. The determinant of a rotation tensor is 1 , and so the motion also preserves relative orientations, as required. In the sequel, we will parameterize the rotation of the body by a set of Euler angles: $v^{1}, v^{2}, v^{3}$. Further, we denote the position vector of the center of mass $\bar{X}$ of the rigid body by $\overline{\mathbf{x}}$ and the position vector of a point (or landmark) $A$ on the rigid body by $\mathbf{x}_{A}$. Both of these position vectors are defined relative to a fixed origin $O$ (cf. Fig. 1).

It is convenient to define two bases for $\mathbb{E}^{3}$ : a fixed right-handed basis $\left\{\mathbf{E}_{1}, \mathbf{E}_{2}, \mathbf{E}_{3}\right\}$ and a corotational (body-fixed) basis $\left\{\mathbf{e}_{1}, \mathbf{e}_{2}, \mathbf{e}_{3}\right\}$. The basis vectors are related: $\mathbf{e}_{i}=\mathbf{Q E}_{i}$, where $i=1,2,3$. For the position vector of the center of mass and the point $A$, we have the representations

$$
\begin{aligned}
\overline{\mathbf{x}} & =X_{1} \mathbf{E}_{1}+X_{2} \mathbf{E}_{2}+X_{3} \mathbf{E}_{3}=x_{1} \mathbf{e}_{1}+x_{2} \mathbf{e}_{2}+x_{3} \mathbf{e}_{3}, \\
\mathbf{x}_{A} & =X_{A_{1}} \mathbf{E}_{1}+X_{A_{2}} \mathbf{E}_{2}+X_{A_{3}} \mathbf{E}_{3}=x_{A_{1}} \mathbf{e}_{1}+x_{A_{2}} \mathbf{e}_{2}+x_{A_{3}} \mathbf{e}_{3} .
\end{aligned}
$$

For any given choice of one of the twelve possible sets of Euler angles, we have the following representation for the angular velocity vector:

$$
\boldsymbol{\omega}=\dot{v}^{1} \mathbf{g}_{1}+\dot{v}^{2} \mathbf{g}_{2}+\dot{v}^{3} \mathbf{g}_{3},
$$

where $\left\{\mathbf{g}_{1}, \mathbf{g}_{2}, \mathbf{g}_{3}\right\}$ is the Euler basis. This set of vectors fails to be a basis at the two singularities experienced by the second Euler angle $v^{2}$.

Following [13-15], the dual Euler basis is defined as the set $\left\{\mathbf{g}^{1}, \mathbf{g}^{2}, \mathbf{g}^{3}\right\}$ such that

$$
\mathbf{g}^{i} \cdot \mathbf{g}_{k}=\delta_{k}^{i}
$$

where $\delta_{k}^{i}$ is the Kronecker delta: $\delta_{k}^{i}=1$ when $i=k$ and is otherwise 0 . We can think of the Euler basis $\left\{\mathbf{g}_{1}, \mathbf{g}_{2}, \mathbf{g}_{3}\right\}$ and the dual Euler basis $\left\{\mathbf{g}^{1}, \mathbf{g}^{2}, \mathbf{g}^{3}\right\}$ as basis vectors in the tangent and cotangent spaces respectively of the manifold $S O(3)$. The connection coefficients associated with the Euler angles are defined as

$$
\gamma_{j k}^{i}=\frac{\partial \mathbf{g}_{j}}{\partial v^{k}} \cdot \mathbf{g}^{i}
$$

Using the identity (4), we can find an alternative representation for the connection coefficients:

$$
\gamma_{j k}^{i}=-\frac{\partial \mathbf{g}^{i}}{\partial v^{k}} \cdot \mathbf{g}_{j}
$$

Further details on the role played by connection coefficients and their relationship to Christoffel symbols can be found in [2, 4].

For a given set of Euler angles, we can compute expressions for the vectors $\mathbf{g}^{k}$ in terms of the bases $\left\{\mathbf{E}_{1}, \mathbf{E}_{2}, \mathbf{E}_{3}\right\}$ :

$$
\mathbf{g}^{k}=\sum_{n=1}^{3} G_{n}^{k} \mathbf{E}_{n} .
$$


The components $G_{i}^{k}$ form a matrix, which we denote by $\mathrm{G}$, and are related to the connection coefficients associated with the Euler angles. Indeed, it is easy to show that

$$
\begin{aligned}
\frac{\partial \mathbf{g}^{k}}{\partial v^{j}} & =\sum_{n=1}^{3} \frac{\partial G_{n}^{k}}{\partial \nu^{j}} \mathbf{E}_{n} \\
& =-\sum_{n=1}^{3} \gamma_{n j}^{k} \mathbf{g}^{n} \\
& =-\sum_{n=1}^{3} \sum_{m=1}^{3} \gamma_{n j}^{k} G_{m}^{n} \mathbf{E}_{m} .
\end{aligned}
$$

Additional details on the derivatives of $\mathbf{g}_{k}$ and $\mathbf{e}_{i}$ can be found in Appendix A. In addition, explicit expressions for these basis vectors, the components $G_{n}^{k}$, and the connection coefficients for the 3-2-1 Euler angles can be found in Appendix B.

\section{Conservative forces and moments}

Motivated by the developments in [15], we assume that the potential energy function $U$ of a rigid body can be expressed as a function of the position vector $\overline{\mathbf{x}}$ and rotation tensor $\mathbf{Q}$. Among others, such a function encompasses the situation where the conservative field is supplied by springs tethering the body to a fixed surface, and a Newtonian gravitational force field attracting the rigid body to a fixed body. Alternatively, we can also express $U$ as a function of the Cartesian coordinates of a point on the rigid body and the Euler angles. In fact, we can readily establish several distinct representations for $U$ :

$$
\begin{aligned}
U & =U(\mathbf{Q}, \overline{\mathbf{x}}) \\
& =U_{1}\left(v^{1}, v^{2}, v^{3}, x_{1}, x_{2}, x_{3}\right)=U_{2}\left(v^{1}, v^{2}, v^{3}, X_{1}, X_{2}, X_{3}\right) \\
& =U_{3}\left(v^{1}, v^{2}, v^{3}, x_{A_{1}}, x_{A_{2}}, x_{A_{3}}\right)=U_{4}\left(v^{1}, v^{2}, v^{3}, X_{A_{1}}, X_{A_{2}}, X_{A_{3}}\right)
\end{aligned}
$$

We obtain $U_{1}$ from $U$ by expressing the components of $\mathbf{Q}$ in terms of the Euler angles and the vector $\overline{\mathbf{x}}$ in terms of its components $x_{k}$ and the bases vectors $\mathbf{e}_{k}: \overline{\mathbf{x}}=\sum_{k=1}^{3} x_{k} \mathbf{e}_{k}$. The vector $\overline{\mathbf{x}}$ can also be expressed in terms of the fixed basis $\left\{\mathbf{E}_{1}, \mathbf{E}_{2}, \mathbf{E}_{3}\right\}$ and this leads to the representation $U_{2}$. Related comments apply for the two potential functions $U_{3,4}$.

To prescribe the conservative force $\mathbf{F}$ and moment (relative to the center of mass) $\mathbf{M}$ acting on the body, we identify the mechanical power of these quantities with the negative of the time rate of change of $U$ :

$$
-\dot{U}=\mathbf{F} \cdot \dot{\overline{\mathbf{x}}}+\mathbf{M} \cdot \boldsymbol{\omega} .
$$

Following [15] and with the help of the dual Euler basis, we find the following representations for $\mathbf{F}$ and $\mathbf{M}$ :

$$
\mathbf{F}=-\sum_{k=1}^{3} \frac{\partial U_{2}}{\partial X_{k}} \mathbf{E}_{k}, \quad \mathbf{M}=-\sum_{k=1}^{3} \frac{\partial U_{2}}{\partial \nu^{k}} \mathbf{g}^{k} .
$$

We emphasize that the force in this expression is assumed to act at the center of mass and the moment $\mathbf{M}$ is taken relative to the center of mass (cf. Fig. 1). 
Now suppose we wish to consider moments relative to other points. There are two cases of primary interest: a point $A$ on the body and a fixed point $O$. With the help of the wellknown identities for the resultant moments relative to $A$ and $O$,

$$
\mathbf{M}_{A}=\mathbf{M}-\left(\mathbf{x}_{A}-\overline{\mathbf{x}}\right) \times \mathbf{F}, \quad \mathbf{M}_{O}=\mathbf{M}+\overline{\mathbf{x}} \times \mathbf{F},
$$

and using the fact that $A$ is a point on the body,

$$
\dot{\mathbf{x}}_{A}=\dot{\overline{\mathbf{x}}}+\omega \times\left(\mathbf{x}_{A}-\overline{\mathbf{x}}\right),
$$

we find that

$$
\mathbf{F} \cdot \dot{\overline{\mathbf{x}}}+\mathbf{M} \cdot \omega=\mathbf{F} \cdot \dot{\mathbf{x}}_{A}+\mathbf{M}_{A} \cdot \omega=\mathbf{F} \cdot(\dot{\overline{\mathbf{x}}}-\omega \times \overline{\mathbf{x}})+\mathbf{M}_{O} \cdot \omega
$$

Invoking (10) and noting that

$$
\dot{\overline{\mathbf{x}}}-\boldsymbol{\omega} \times \overline{\mathbf{x}}=\sum_{k=1}^{3} \dot{x}_{k} \mathbf{e}_{k},
$$

we conclude that

$$
\mathbf{F}=-\sum_{k=1}^{3} \frac{\partial U_{4}}{\partial X_{A_{k}}} \mathbf{E}_{k}, \quad \mathbf{M}_{A}=-\sum_{k=1}^{3} \frac{\partial U_{4}}{\partial \nu^{k}} \mathbf{g}^{k},
$$

and

$$
\mathbf{F}=-\sum_{k=1}^{3} \frac{\partial U_{1}}{\partial x_{k}} \mathbf{e}_{k}, \quad \mathbf{M}_{O}=-\sum_{k=1}^{3} \frac{\partial U_{1}}{\partial \nu^{k}} \mathbf{g}^{k} .
$$

The contrast between (11) and (17) is illuminating. When moments about a fixed point $O$ are considered, the natural representation for the conservative force is with respect to the corotational basis. This is in surprising contrast to the case where the moments are taken relative to a material point on the body.

\section{The Cartesian stiffness matrix}

For any of the representations of the conservative forces and moments, a Cartesian stiffness matrix can be defined. This matrix relates the changes to Cartesian components of a pair of forces and moments in two configurations of the rigid body to the Cartesian components of the infinitesimal displacement of a point on the rigid body and the infinitesimal rotation between the configurations. In experimental situations, it is often easier to measure $\mathbf{F} \cdot \mathbf{E}_{i}$ and $\mathbf{M}_{O} \cdot \mathbf{E}_{i}$ using a load cell than the $\mathbf{e}_{r}$ and $\mathbf{g}^{k}$ components featuring in (17). An example of this situation arises in experiments conducted on the lumbar spine using a servo-hydraulic test frame (see, e.g., [16]).

Additionally, as mentioned in [9], the Cartesian matrix is particularly useful in mechanisms such as robots where motion is parameterized in terms of small rotations about and small translations along the axes of a reference frame. In these instances, the coordinate system of the reference frame rather than generalized coordinates is the natural choice of parameterization to use. 


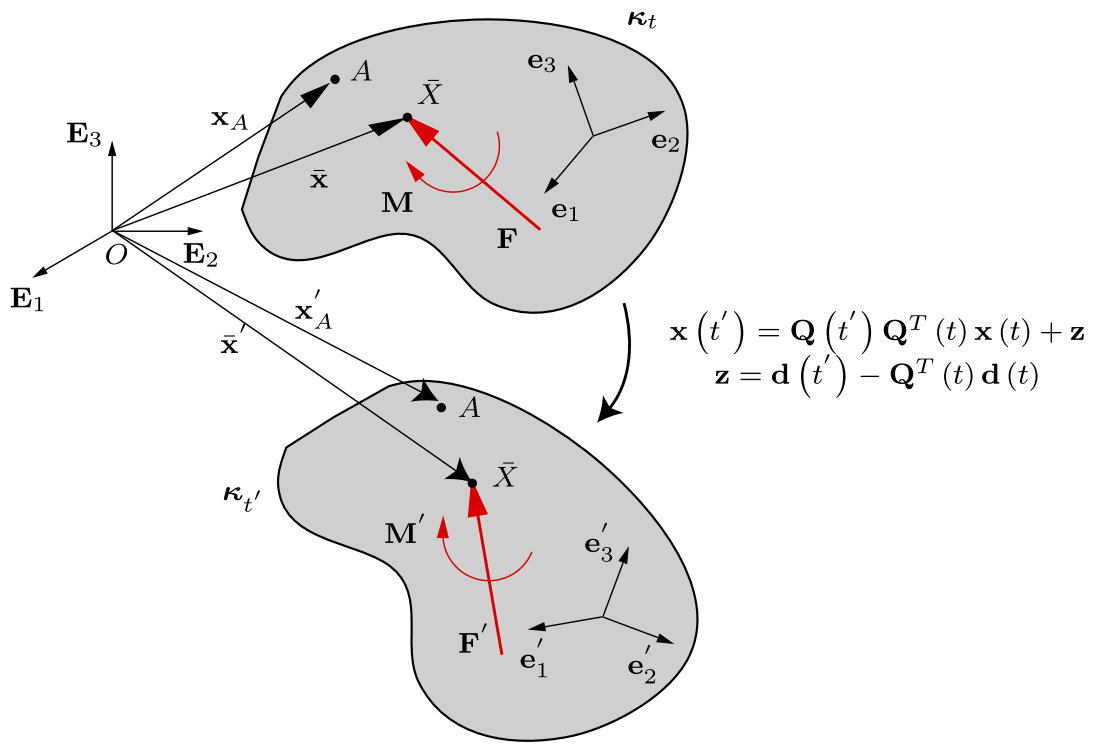

Fig. 2 Two configurations $\kappa_{t}$ and $\kappa_{t^{\prime}}$ of a rigid body $\mathcal{B}$. The kinematic quantities associated with the configuration $\kappa_{t^{\prime}}$ are distinguished by a superscript ${ }^{\prime}$ from those associated with the configuration $\kappa_{t}$ : e.g., $\overline{\mathbf{x}}^{\prime}=\overline{\mathbf{x}}\left(t^{\prime}\right)$

To elaborate, consider two configurations of a rigid body $\kappa_{t}$ and $\kappa_{t^{\prime}}$. We distinguish quantities associated with $\boldsymbol{\kappa}_{t^{\prime}}$ with a superscript ${ }^{\prime}$. The motion between these configurations can be defined with the help of (1):

$$
\mathbf{x}^{\prime}=\mathbf{x}\left(t^{\prime}\right)=\mathbf{Q}\left(t^{\prime}\right) \mathbf{Q}^{T}(t) \mathbf{x}(t)+\mathbf{z}, \quad \mathbf{z}=\mathbf{d}\left(t^{\prime}\right)-\mathbf{Q}^{T}(t) \mathbf{d}(t)
$$

We shall assume that the two configurations differ by an infinitesimal rigid body motion. Thus,

$$
\Delta \overline{\mathbf{x}}=\overline{\mathbf{x}}^{\prime}-\overline{\mathbf{x}}=O(\epsilon), \quad \mathbf{I}+\Delta \mathbf{Q}=\mathbf{Q}\left(t^{\prime}\right) \mathbf{Q}^{T}(t), \quad \Delta \mathbf{Q}=O(\epsilon),
$$

where $\epsilon$ is a small number and $\mathbf{I}$ is the identity tensor.

As the rotation $\Delta \mathbf{Q}$ is infinitesimal, $\Delta \mathbf{Q}$ is skew-symmetric [20]. If $v^{k^{\prime}}$ denote the values of the Euler angles associated with $\mathbf{Q}\left(t^{\prime}\right)$, then a lengthy, but straightforward calculation shows that the axial vector $\Delta \boldsymbol{\theta}$ of $\Delta \mathbf{Q}$ has the representation (cf. (117))

$$
\Delta \boldsymbol{\theta}=\sum_{k=1}^{3}\left(v^{k^{\prime}}-v^{k}\right) \mathbf{g}_{k}+O\left(\epsilon^{2}\right),
$$

where $\mathbf{g}_{k}$ are the Euler basis vectors associated with $\mathbf{Q}(t)$. It follows that

$$
v^{k^{\prime}}-v^{k}=\Delta \boldsymbol{\theta} \cdot \mathbf{g}^{k}=\sum_{i=1}^{3} G_{i}^{k} \Delta \boldsymbol{\theta} \cdot \mathbf{E}_{i},
$$

where we used (7) to express the dual Euler basis vectors in terms of the Cartesian basis vectors. 
To first order in $\epsilon$, the displacement vector $\Delta \overline{\mathbf{x}}$ has the representations

$$
\Delta \overline{\mathbf{x}}=\sum_{k=1}^{3}\left(X_{k}^{\prime}-X_{k}\right) \mathbf{E}_{k}=\sum_{r=1}^{3}\left(x_{r}^{\prime}-x_{r}\right) \mathbf{e}_{r}+\Delta \boldsymbol{\theta} \times \overline{\mathbf{x}},
$$

where $x_{k}^{\prime}=\overline{\mathbf{x}}^{\prime} \cdot \mathbf{e}_{k}^{\prime}=\overline{\mathbf{x}}^{\prime} \cdot \mathbf{e}_{k}+\overline{\mathbf{x}}^{\prime} \cdot\left(\Delta \mathbf{Q} \mathbf{e}_{k}\right)$. Consequently,

$$
\begin{aligned}
X_{k}^{\prime}-X_{k} & =\Delta \overline{\mathbf{x}} \cdot \mathbf{E}_{k}, \\
x_{r}^{\prime}-x_{r} & =\Delta \overline{\mathbf{x}} \cdot\left(\sum_{k=1}^{3} Q_{r k} \mathbf{E}_{k}\right)+\Delta \boldsymbol{\theta} \cdot\left(\mathbf{e}_{r} \times \overline{\mathbf{x}}\right) .
\end{aligned}
$$

The presence of the term $\Delta \boldsymbol{\theta} \cdot\left(\mathbf{e}_{r} \times \overline{\mathbf{x}}\right)$ in $(23)_{2}$ reflects the difference in the vectors $\mathbf{e}_{r}^{\prime}$ and $\mathbf{e}_{r}$.

We are now in a position to define a Cartesian stiffness matrix $\mathrm{K}_{c}$. Based on the four functions discussed earlier, there are four possible matrices and we distinguish them by a left subscript. All of the stiffness matrices are obtained by performing a Taylor series expansion of the expressions for the appropriate conservative forces and moments. In addition, the developments for the stiffness matrices associated with the potential energies $U_{3}$ and $U_{4}$ are similar to those presented for $U_{1}$ and $U_{2}$, respectively. In the interests of brevity, they are omitted.

\subsection{The stiffness matrix ${ }_{1} \mathrm{~K}_{c}$}

The first Cartesian stiffness matrix, which we denote by ${ }_{1} \mathrm{~K}_{c}$, relates the differences in the force $\mathbf{F}$ and moment $\mathbf{M}_{O}$ in the configurations $\boldsymbol{\kappa}_{t^{\prime}}$ and $\boldsymbol{\kappa}_{t}$ to the infinitesimal displacement vectors $\Delta \overline{\mathbf{x}}$ and $\Delta \boldsymbol{\theta}$. The matrix ${ }_{1} \mathrm{~K}_{c}$ is defined by the identity

$$
\Delta \mathrm{F}=-{ }_{1} \mathrm{~K}_{c} \Delta \mathrm{x}+O\left(\epsilon^{2}\right),
$$

where

$$
\Delta \mathrm{F}=\left[\begin{array}{c}
\left(\mathbf{F}^{\prime}-\mathbf{F}\right) \cdot \mathbf{E}_{1} \\
\left(\mathbf{F}^{\prime}-\mathbf{F}\right) \cdot \mathbf{E}_{2} \\
\left(\mathbf{F}^{\prime}-\mathbf{F}\right) \cdot \mathbf{E}_{3} \\
\left(\mathbf{M}_{O}^{\prime}-\mathbf{M}_{O}\right) \cdot \mathbf{E}_{1} \\
\left(\mathbf{M}_{O}^{\prime}-\mathbf{M}_{O}\right) \cdot \mathbf{E}_{2} \\
\left(\mathbf{M}_{O}^{\prime}-\mathbf{M}_{O}\right) \cdot \mathbf{E}_{3}
\end{array}\right], \quad \Delta \mathrm{x}=\left[\begin{array}{c}
\Delta \overline{\mathbf{x}} \cdot \mathbf{E}_{1} \\
\Delta \overline{\mathbf{x}} \cdot \mathbf{E}_{2} \\
\Delta \overline{\mathbf{x}} \cdot \mathbf{E}_{3} \\
\Delta \boldsymbol{\theta} \cdot \mathbf{E}_{1} \\
\Delta \boldsymbol{\theta} \cdot \mathbf{E}_{2} \\
\Delta \boldsymbol{\theta} \cdot \mathbf{E}_{3}
\end{array}\right], \quad \Delta \mathrm{s}=\left[\begin{array}{c}
(\Delta \overline{\mathbf{x}}-\Delta \boldsymbol{\theta} \times \overline{\mathbf{x}}) \cdot \mathbf{E}_{1} \\
(\Delta \overline{\mathbf{x}}-\Delta \boldsymbol{\theta} \times \overline{\mathbf{x}}) \cdot \mathbf{E}_{2} \\
(\Delta \overline{\mathbf{x}}-\Delta \boldsymbol{\theta} \times \overline{\mathbf{x}}) \cdot \mathbf{E}_{3} \\
\Delta \boldsymbol{\theta} \cdot \mathbf{E}_{1} \\
\Delta \boldsymbol{\theta} \cdot \mathbf{E}_{2} \\
\Delta \boldsymbol{\theta} \cdot \mathbf{E}_{3}
\end{array}\right]
$$

We have also taken this opportunity to define another displacement vector $\Delta \mathrm{s}$ in order to be able to compare our work with those of Ciblak and Lipkin [5] and others who use screw theory.

To obtain a representation for ${ }_{1} \mathrm{~K}_{c}$, we perform Taylor series expansions of the expressions for $\mathbf{F}$ and $\mathbf{M}_{O}$ about the configuration $\boldsymbol{\kappa}_{t}$ (cf. (17)). After ignoring terms of order $\epsilon^{2}$, we find that

$$
\begin{aligned}
\mathbf{F}^{\prime}-\mathbf{F} & =-\sum_{k=1}^{3} \sum_{i=1}^{3} \frac{\partial}{\partial x_{k}}\left(\frac{\partial U_{1}}{\partial x_{i}} \mathbf{e}_{i}\right)\left(x_{k}^{\prime}-x_{k}\right)-\sum_{k=1}^{3} \frac{\partial}{\partial v^{k}}\left(\sum_{i=1}^{3} \frac{\partial U_{1}}{\partial x_{i}} \mathbf{e}_{i}\right)\left(v^{k^{\prime}}-v^{k}\right), \\
\mathbf{M}_{O}^{\prime}-\mathbf{M}_{O} & =-\sum_{k=1}^{3} \frac{\partial}{\partial x_{k}}\left(\sum_{i=1}^{3} \frac{\partial U_{1}}{\partial v^{i}} \mathbf{g}^{i}\right)\left(x_{k}^{\prime}-x_{k}\right)-\sum_{k=1}^{3} \frac{\partial}{\partial \nu^{k}}\left(\sum_{i=1}^{3} \frac{\partial U_{1}}{\partial v^{i}} \mathbf{g}^{i}\right)\left(v^{k^{\prime}}-v^{k}\right) .
\end{aligned}
$$


Performing some rearranging and using (21) and $(23)_{2},(26)$ can be rewritten as

$$
\begin{aligned}
\mathbf{F}^{\prime}-\mathbf{F}= & -\sum_{i=1}^{3}\left(\sum_{r=1}^{3} \sum_{k=1}^{3}\left(\frac{\partial^{2} U_{1}}{\partial x_{k} \partial x_{i}}\right) Q_{k r} \Delta \overline{\mathbf{x}} \cdot \mathbf{E}_{r}+\sum_{k=1}^{3} \sum_{r=1}^{3}\left(\frac{\partial^{2} U_{1}}{\partial \nu^{k} \partial x_{i}}\right) G_{r}^{k} \Delta \boldsymbol{\theta} \cdot \mathbf{E}_{r}\right) \mathbf{e}_{i} \\
& -\sum_{k=1}^{3} \sum_{i=1}^{3} \sum_{r=1}^{3}\left(\frac{\partial U_{1}}{\partial x_{i}}\right)\left(G_{r}^{k} \Delta \boldsymbol{\theta} \cdot \mathbf{E}_{r}\right) \frac{\partial \mathbf{e}_{i}}{\partial \nu^{k}} \\
& -\sum_{i=1}^{3} \sum_{k=1}^{3}\left(\frac{\partial^{2} U_{1}}{\partial x_{k} \partial x_{i}}\right)\left(\Delta \theta \cdot\left(\mathbf{e}_{k} \times \overline{\mathbf{x}}\right)\right) \mathbf{e}_{i}, \\
\mathbf{M}_{O}^{\prime}-\mathbf{M}_{O}= & -\sum_{i=1}^{3}\left(\sum_{r=1}^{3} \sum_{k=1}^{3}\left(\frac{\partial^{2} U_{1}}{\partial x_{k} \partial v^{i}}\right) Q_{k r} \Delta \overline{\mathbf{x}} \cdot \mathbf{E}_{r}+\sum_{r=1}^{3} \sum_{k=1}^{3}\left(\frac{\partial^{2} U_{1}}{\partial \nu^{k} \partial v^{i}}\right) G_{r}^{k} \Delta \boldsymbol{\theta} \cdot \mathbf{E}_{r}\right) \mathbf{g}^{i} \\
& -\sum_{k=1}^{3} \sum_{i=1}^{3} \sum_{r=1}^{3}\left(\frac{\partial U_{1}}{\partial \nu^{i}}\right)\left(G_{r}^{k} \Delta \boldsymbol{\theta} \cdot \mathbf{\mathbf { E } _ { r }}\right) \frac{\partial \mathbf{g}^{i}}{\partial \nu^{k}} \\
& -\sum_{i=1}^{3} \sum_{k=1}^{3}\left(\frac{\partial^{2} U_{1}}{\partial x_{k} \partial \nu^{i}}\right)\left(\Delta \theta \cdot\left(\mathbf{e}_{k} \times \overline{\mathbf{x}}\right)\right) \mathbf{g}^{i} .
\end{aligned}
$$

In (27), the components of the matrix $\mathrm{Q}$ are $Q_{i k}=\mathbf{e}_{i}(t) \cdot \mathbf{E}_{k}$ and the components of the matrix $\mathrm{G}$ are $G_{k}^{i}=\mathbf{g}^{i}(t) \cdot \mathbf{E}_{k}$.

Taking the $\mathbf{E}_{n}$ components of the force and moment vectors in (27) and using (8) and (120), the following representation for the stiffness matrix is obtained:

$$
{ }_{1} \mathrm{~K}_{c}=\left[\begin{array}{cc}
\mathrm{Q}^{T} & 0 \\
0 & \mathrm{G}^{T}
\end{array}\right]{ }_{1} \mathrm{H}\left[\begin{array}{cc}
\mathrm{Q} & 0 \\
0 & \mathrm{G}
\end{array}\right]+\left[\begin{array}{ll}
0 & { }_{1} \mathrm{C} \\
0 & { }_{1} \mathrm{D}
\end{array}\right]+\left[\begin{array}{ll}
0 & { }_{1} \mathrm{Y} \\
0 & { }_{1} \mathrm{Z}
\end{array}\right] .
$$

Here, ${ }_{1} \mathrm{H}$ is the Hessian of the potential energy function $U_{1}$ :

$$
{ }_{1} \mathrm{H}=\left[\begin{array}{ll}
{ }_{1} \mathrm{~K}_{1} & { }_{1} \mathrm{~K}_{3} \\
{ }_{1} \mathrm{~K}_{3}^{T} & { }_{1} \mathrm{~K}_{2}
\end{array}\right]
$$

with

$$
{ }_{1} \mathrm{~K}_{1, i j}=\frac{\partial^{2} U_{1}}{\partial x_{i} \partial x_{j}}, \quad{ }_{1} \mathrm{~K}_{2, i j}=\frac{\partial^{2} U_{1}}{\partial \nu^{i} \partial \nu^{j}}, \quad{ }_{1} \mathrm{~K}_{3, i j}=\frac{\partial^{2} U_{1}}{\partial \nu^{i} \partial x_{j}} .
$$

The components of the $3 \times 3$ matrices ${ }_{1} \mathrm{C},{ }_{1} \mathrm{D},{ }_{1} \mathrm{Y}$, and ${ }_{1} \mathrm{Z}$ are, respectively,

$$
\begin{aligned}
{ }_{1} C_{m n} & =\sum_{k=1}^{3} \sum_{i=1}^{3} \frac{\partial Q_{i m}}{\partial v^{k}} G_{n}^{k} \frac{\partial U_{1}}{\partial x_{i}} \\
{ }_{1} D_{m n} & =\sum_{k=1}^{3} \sum_{i=1}^{3} \frac{\partial G_{m}^{i}}{\partial \nu^{k}} G_{n}^{k} \frac{\partial U_{1}}{\partial \nu^{i}} \\
{ }_{1} \mathrm{Y} & =\mathrm{Q}^{T}{ }_{1} \mathrm{~K}_{1} \mathrm{SQ}, \\
{ }_{1} \mathrm{Z} & =\mathrm{G}^{T}{ }_{1} \mathrm{~K}_{3}^{T} \mathrm{SQ},
\end{aligned}
$$


and the components of the skew-symmetric matrix $S$ are

$$
S_{m n}=\overline{\mathbf{x}} \cdot\left(\mathbf{e}_{n} \times \mathbf{e}_{m}\right) .
$$

We emphasize that the partial derivatives and vectors in the expressions for the components of ${ }_{1} \mathrm{~K}_{c}$ are all evaluated using the values $\overline{\mathbf{x}}$ and $v^{k}$ associated with the configuration $\boldsymbol{\kappa}_{t}$. It will be shown in Sect. 5 that ${ }_{1} \mathbf{C}$ is skew-symmetric and has an axial vector $\mathbf{F} \cdot \mathbf{E}_{i}$, while the skew-symmetric part of ${ }_{1} \mathrm{D}$ has an axial vector $\frac{1}{2} \mathbf{M}_{O} \cdot \mathbf{E}_{i}$.

4.2 The stiffness matrix ${ }_{2} \mathrm{~K}_{c}$

A second Cartesian stiffness matrix can be defined relating the components of $\mathbf{F} \cdot \mathbf{E}_{k}$ and $\mathbf{M} \cdot \mathbf{E}_{k}$ to the vector $\Delta \mathbf{x}$ :

$$
\Delta \overline{\mathrm{F}}=-{ }_{2} \mathrm{~K}_{c} \Delta \mathrm{x}+O\left(\epsilon^{2}\right),
$$

where

$$
\Delta \overline{\mathrm{F}}=\left[\begin{array}{c}
\left(\mathbf{F}^{\prime}-\mathbf{F}\right) \cdot \mathbf{E}_{1} \\
\left(\mathbf{F}^{\prime}-\mathbf{F}\right) \cdot \mathbf{E}_{2} \\
\left(\mathbf{F}^{\prime}-\mathbf{F}\right) \cdot \mathbf{E}_{3} \\
\left(\mathbf{M}^{\prime}-\mathbf{M}\right) \cdot \mathbf{E}_{1} \\
\left(\mathbf{M}^{\prime}-\mathbf{M}\right) \cdot \mathbf{E}_{2} \\
\left(\mathbf{M}^{\prime}-\mathbf{M}\right) \cdot \mathbf{E}_{3}
\end{array}\right] .
$$

The derivation of ${ }_{2} \mathrm{~K}_{c}$ closely follows the developments in the previous subsection and they are omitted in the interest of brevity. In summary, we find that

$$
{ }_{2} \mathrm{~K}_{c}=\left[\begin{array}{cc}
\mathrm{I} & 0 \\
0 & \mathrm{G}^{T}
\end{array}\right]{ }_{2} \mathrm{H}\left[\begin{array}{ll}
\mathrm{I} & 0 \\
0 & \mathrm{G}
\end{array}\right]+\left[\begin{array}{cc}
0 & 0 \\
0 & { }_{2} \mathrm{D}
\end{array}\right] .
$$

Here, ${ }_{2} \mathrm{H}$ is the Hessian of the potential energy function $U_{2}$ :

$$
{ }_{2} \mathrm{H}=\left[\begin{array}{ll}
{ }_{2} \mathrm{~K}_{1} & { }_{2} \mathrm{~K}_{3} \\
{ }_{2} \mathrm{~K}_{3}^{T} & { }_{2} \mathrm{~K}_{2}
\end{array}\right],
$$

with

$$
{ }_{2} \mathrm{~K}_{1, i j}=\frac{\partial^{2} U_{2}}{\partial X_{i} \partial X_{j}}, \quad{ }_{2} \mathrm{~K}_{2, i j}=\frac{\partial^{2} U_{2}}{\partial \nu^{i} \partial v^{j}}, \quad{ }_{2} \mathrm{~K}_{3, i j}=\frac{\partial^{2} U_{2}}{\partial \nu^{i} \partial X_{j}},
$$

and

$$
{ }_{2} D_{m n}=\sum_{k=1}^{3} \sum_{i=1}^{3} \frac{\partial G_{m}^{i}}{\partial \nu^{k}} G_{n}^{k} \frac{\partial U_{2}}{\partial \nu^{i}} .
$$

In the previous expression, ${ }_{2} D_{m n}$ are the components of the $3 \times 3$ matrix ${ }_{2} \mathrm{D}$. It will be shown in Sect. 5 that the skew-symmetric part of ${ }_{2} \mathrm{D}$ has an axial vector $\frac{1}{2} \mathbf{M} \cdot \mathbf{E}_{i}$.

\subsection{Remarks}

As noted by several authors (e.g., [10]), it is important to distinguish the Cartesian stiffness matrix $\mathrm{K}_{c}$ from the stiffness matrix or Hessian $\mathrm{H}$ in analytical dynamics. Indeed, it is transparent from (28) and (35), how $\mathrm{K}_{c}$ is a function of, and distinct from $\mathrm{H}$. 
The matrices ${ }_{1} \mathrm{~K}_{c}$ and ${ }_{2} \mathrm{~K}_{c}$ both provide expressions for $\mathbf{F}^{\prime}-\mathbf{F}$ in terms of $\Delta \overline{\mathbf{x}}$ and $\Delta \boldsymbol{\theta}$. Consequently, 18 of the 36 components of ${ }_{1} \mathrm{~K}_{c}$ and ${ }_{2} \mathrm{~K}_{c}$ are identical:

$$
\mathrm{Q}^{T}{ }_{1} \mathrm{~K}_{1} \mathrm{Q}={ }_{2} \mathrm{~K}_{1}, \quad \mathrm{Q}^{T}{ }_{1} \mathrm{~K}_{3} \mathrm{G}+{ }_{1} \mathrm{C}+{ }_{1} \mathrm{Y}={ }_{2} \mathrm{~K}_{3} \mathrm{G} \text {. }
$$

This result will be used in Sect. 7 to validate our numerical computations of ${ }_{1} \mathrm{~K}_{c}$ and ${ }_{2} \mathrm{~K}_{c}$ for a specific mechanism.

Now suppose we were to consider a point $P$ which is not a material point of the body. In this case, $\mathbf{v}_{P} \neq \dot{\overline{\mathbf{x}}}+\omega \times\left(\mathbf{x}_{P}-\overline{\mathbf{x}}\right)$. Unless $P$ is a fixed point, it is not possible to establish identities of the form (14) featuring $\mathbf{F}$ and the resultant moment relative to $P, \mathbf{M}_{P}$. As a result, if we wish to establish an expression for a stiffness matrix relative a point $P$ on the helical axis of motion, we would need to consider a fixed point $O$ which instantaneously coincides with the point $P$ of interest. The stiffness matrix would then be ${ }_{1} \mathrm{~K}_{c}$. If $P$ were to move, then we would need to relocate $O$, recompute $\overline{\mathbf{x}} \cdot \mathbf{e}_{i}$ and reevaluate ${ }_{1} \mathrm{~K}_{c}$.

In the work of Ciblak and Lipkin [5] and others where screw theory is used, the incremental displacement vector $\Delta \mathrm{s}$ is used instead of $\Delta \mathrm{x}$ (cf. (25)). This choice of displacement leads to another stiffness matrix, which we denote by $\mathrm{K}_{O}$ is defined:

$$
\Delta \mathrm{F}=-\mathrm{K}_{O} \Delta \mathrm{s}+O\left(\epsilon^{2}\right),
$$

Here, $\Delta \mathrm{s}$ is defined by $(25)_{3}$. Paralleling the developments which lead to (28), we find the following representation for $\mathrm{K}_{O}$ :

$$
\mathrm{K}_{O}=\left[\begin{array}{cc}
\mathrm{Q}^{T} & 0 \\
0 & \mathrm{G}^{T}
\end{array}\right]{ }_{1} \mathrm{H}\left[\begin{array}{cc}
\mathrm{Q} & 0 \\
0 & \mathrm{G}
\end{array}\right]+\left[\begin{array}{ll}
0 & { }_{1} \mathrm{C} \\
0 & { }_{1} \mathrm{D}
\end{array}\right] .
$$

That is,

$$
{ }_{1} \mathrm{~K}_{c}=\mathrm{K}_{O}+\left[\begin{array}{ll}
0 & 1 \\
0 & \mathrm{Y} \\
0
\end{array}\right],
$$

and so ${ }_{1} \mathrm{~K}_{c}$ can be asymmetric even when $\mathrm{K}_{O}$ is symmetric.

The stiffness matrices in (28) and (35) relate the $\mathbf{E}_{i}$ components of the infinitesimal displacements to the increments in the $\mathbf{E}_{i}$ components of the conservative forces and moments. It is possible to define another Cartesian stiffness matrix where the $\mathbf{E}_{i}$ components are replaced by the components with respect to $\mathbf{e}_{i}(t)$. As an example,

$$
\left[\begin{array}{c}
\left(\mathbf{F}^{\prime}-\mathbf{F}\right) \cdot \mathbf{e}_{1}(t) \\
\left(\mathbf{F}^{\prime}-\mathbf{F}\right) \cdot \mathbf{e}_{2}(t) \\
\left(\mathbf{F}^{\prime}-\mathbf{F}\right) \cdot \mathbf{e}_{3}(t) \\
\left(\mathbf{M}_{O}^{\prime}-\mathbf{M}_{O}\right) \cdot \mathbf{e}_{1}(t) \\
\left(\mathbf{M}_{O}^{\prime}-\mathbf{M}_{O}\right) \cdot \mathbf{e}_{2}(t) \\
\left(\mathbf{M}_{O}^{\prime}-\mathbf{M}_{O}\right) \cdot \mathbf{e}_{3}(t)
\end{array}\right]=-\left[\begin{array}{ll}
\mathbf{Q} & 0 \\
0 & \mathrm{Q}
\end{array}\right]{ }_{1} \mathrm{~K}_{c}\left[\begin{array}{cc}
\mathrm{Q}^{T} & 0 \\
0 & \mathrm{Q}^{T}
\end{array}\right]\left[\begin{array}{c}
\Delta \overline{\mathbf{x}} \cdot \mathbf{e}_{1}(t) \\
\Delta \overline{\mathbf{x}} \cdot \mathbf{e}_{2}(t) \\
\Delta \overline{\mathbf{x}} \cdot \mathbf{e}_{3}(t) \\
\Delta \boldsymbol{\theta} \cdot \mathbf{e}_{1}(t) \\
\Delta \boldsymbol{\theta} \cdot \mathbf{e}_{2}(t) \\
\Delta \boldsymbol{\theta} \cdot \mathbf{e}_{3}(t)
\end{array}\right] .
$$

From this equation, it is easy to infer that the Cartesian stiffness matrix in this case is a transformation of the stiffness matrix when the fixed basis is used.

\section{The asymmetric parts of the stiffness matrices}

As is the case with the situations discussed in $[5,8,9,21]$, the asymmetry of $\mathrm{K}_{O}$ arises because of the presence of a nonzero gradient of $U$ for the configuration $\kappa_{t}$. A similar 
situation arises for ${ }_{1} \mathrm{~K}_{c}$ and ${ }_{2} \mathrm{~K}_{c}$. For ${ }_{1} \mathrm{~K}_{c}$, the nonzero gradient of $U_{1}$ then combines with the dependency of the basis vectors $\mathbf{e}_{k}$ and $\mathbf{g}^{k}$ on the Euler angles to yield asymmetric contributions to the Cartesian stiffness matrix. On the other hand, for ${ }_{2} \mathrm{~K}_{c}$ only the moment $\mathbf{M}$ contributes to the asymmetry of this matrix. If the body is in equilibrium under the sole action of conservative forces and moments in the configuration $\boldsymbol{\kappa}_{t}$, then the gradient of $U$ will be zero. In this case, the stiffness matrices $\mathrm{K}_{O}$ and ${ }_{2} \mathrm{~K}_{c}$ will be symmetric, however the matrix ${ }_{1} \mathrm{~K}_{c}$ may still be asymmetric (due to the presence of non-zero ${ }_{1} \mathrm{Y}$ and ${ }_{1} Z$ ).

As mentioned, the asymmetry of $\mathrm{K}_{O}$ and part of the asymmetry of ${ }_{1} \mathrm{~K}_{c}$ is due to the presence of the matrices ${ }_{1} C$ and ${ }_{1} D$. These two matrices have several unusual features. In particular, ${ }_{1} \mathrm{C}$ is skew-symmetric, and the skew-symmetric parts of ${ }_{1} \mathrm{C}$ and ${ }_{1} \mathrm{D}$ can be related to the force $\mathbf{F}$ and moment $\mathbf{M}_{O}$, respectively. Our results for the matrix $\mathrm{K}_{O}$ in this respect are the analogues of Theorem 1 of Ciblak and Lipkin [5], Corollary 1 in Howard et al. [9], and Proposition 4.2 in Žefran and Kumar [21]. In a similar manner, for the stiffness matrix ${ }_{2} \mathrm{~K}_{c}$, the skew-symmetric part of ${ }_{2} \mathrm{D}$ is related to the moment $\mathbf{M}$.

We start with the matrix ${ }_{1} \mathrm{C}$. This matrix has a strong dependency on the change in the corotational basis vectors with respect to the Euler angles: $\frac{\partial \mathbf{e}_{i}}{\partial v^{k}}$. To prove the skew-symmetry of ${ }_{1} \mathrm{C}$, we first observe that the components of ${ }_{1} \mathrm{C}$ can be used to form a tensor ${ }_{1} \mathbf{C}$ :

$$
{ }_{1} \mathbf{C}=\sum_{m=1}^{3} \sum_{n=1}^{3}{ }_{1} C_{m n} \mathbf{E}_{m} \otimes \mathbf{E}_{n}=\sum_{i=1}^{3} \sum_{k=1}^{3} \frac{\partial U_{1}}{\partial x_{i}} \frac{\partial \mathbf{e}_{i}}{\partial \nu^{k}} \otimes \mathbf{g}^{k},
$$

where $\otimes$ is the tensor product of two vectors: $(\mathbf{a} \otimes \mathbf{b}) \mathbf{c}=\mathbf{a}(\mathbf{b} \cdot \mathbf{c})$ for all vectors $\mathbf{a}, \mathbf{b}$, and $\mathbf{c}$. We now invoke two identities (cf. (17) ${ }_{1}$ and (119)):

$$
\frac{\partial \mathbf{e}_{i}}{\partial \nu^{k}}=\mathbf{g}_{k} \times \mathbf{e}_{i}, \quad \frac{\partial U_{1}}{\partial x_{i}}=-\mathbf{F} \cdot \mathbf{e}_{i} .
$$

Thus,

$$
{ }_{1} \mathbf{C}=\sum_{k=1}^{3}\left(\mathbf{g}_{k} \times\left(\sum_{i=1}^{3} \frac{\partial U_{1}}{\partial x_{i}} \mathbf{e}_{i}\right)\right) \otimes \mathbf{g}^{k}=-\sum_{k=1}^{3}\left(\mathbf{g}_{k} \times \mathbf{F}\right) \otimes \mathbf{g}^{k} .
$$

A direct calculation shows that, for any vector $\mathbf{a}$,

$$
-\left(\sum_{k=1}^{3}\left(\mathbf{g}_{k} \times \mathbf{F}\right) \otimes \mathbf{g}^{k}\right) \mathbf{a}=\mathbf{F} \times \mathbf{a} .
$$

Thus, we conclude that the matrix ${ }_{1} \mathrm{C}$ is skew-symmetric and that

$$
{ }_{1} C_{32}=-{ }_{1} C_{23}=\mathbf{F} \cdot \mathbf{E}_{1}, \quad{ }_{1} C_{13}=-{ }_{1} C_{31}=\mathbf{F} \cdot \mathbf{E}_{2}, \quad{ }_{1} C_{21}=-{ }_{1} C_{12}=\mathbf{F} \cdot \mathbf{E}_{3} .
$$

This result is a generalization of Theorem 1 of Ciblak and Lipkin [5] to systems where the elastic element can also supply pure moments.

It is tempting to conclude that ${ }_{1} \mathrm{D}$ will also be skew-symmetric, but this is not the case. The skew-symmetric part of this matrix is in direct correspondence with the components $\mathbf{M}_{O} \cdot \mathbf{E}_{k}$. To arrive at this result, we note that the components of ${ }_{1} \mathrm{D}$ can be used to form a tensor ${ }_{1} \mathbf{D}$ :

$$
{ }_{1} \mathbf{D}=\sum_{m=1}^{3} \sum_{n=1}^{3}{ }_{1} D_{m n} \mathbf{E}_{m} \otimes \mathbf{E}_{n}=\sum_{i=1}^{3} \sum_{k=1}^{3} \frac{\partial U_{1}}{\partial \nu^{i}} \frac{\partial \mathbf{g}^{i}}{\partial \nu^{k}} \otimes \mathbf{g}^{k}
$$


With the help of (8), we can express the derivatives of $\mathbf{g}^{i}$ using the connection coefficients:

$$
{ }_{1} \mathbf{D}=-\sum_{i=1}^{3} \sum_{j=1}^{3} \sum_{k=1}^{3} \frac{\partial U_{1}}{\partial \nu^{i}} \gamma_{j k}^{i} \mathbf{g}^{j} \otimes \mathbf{g}^{k} .
$$

However, as (cf. (5) and (17)2),

$$
\sum_{i=1}^{3} \gamma_{j k}^{i} \mathbf{g}_{i}=\frac{\partial \mathbf{g}_{j}}{\partial \nu^{k}}, \quad \mathbf{M}_{O} \cdot \mathbf{g}_{i}=-\frac{\partial U_{1}}{\partial \nu^{i}},
$$

we find that the expression for ${ }_{1} \mathbf{D}$ simplifies to

$$
{ }_{1} \mathbf{D}=\sum_{k=1}^{3} \sum_{j=1}^{3}\left(\mathbf{M}_{O} \cdot \frac{\partial \mathbf{g}_{j}}{\partial \nu^{k}}\right) \mathbf{g}^{j} \otimes \mathbf{g}^{k} .
$$

Thus,

$$
{ }_{1} \mathbf{D}-{ }_{1} \mathbf{D}^{T}=\sum_{k=1}^{3} \sum_{j=1}^{3}\left(\mathbf{M}_{O} \cdot\left(\frac{\partial \mathbf{g}_{j}}{\partial \nu^{k}}-\frac{\partial \mathbf{g}_{k}}{\partial \nu^{j}}\right)\right) \mathbf{g}^{j} \otimes \mathbf{g}^{k} .
$$

We next appeal to the identity (123) and conclude that

$$
{ }_{1} \mathbf{D}-{ }_{1} \mathbf{D}^{T}=\sum_{k=1}^{3} \sum_{j=1}^{3}\left(\mathbf{M}_{O} \cdot\left(\mathbf{g}_{k} \times \mathbf{g}_{j}\right)\right) \mathbf{g}^{j} \otimes \mathbf{g}^{k} .
$$

To compute the axial vector of this tensor, we note that, for any vector $\mathbf{a}=\sum_{r=1}^{3} a^{r} \mathbf{g}_{r}$,

$$
\begin{aligned}
\left(\sum_{k=1}^{3} \sum_{j=1}^{3}\left(\mathbf{M}_{O} \cdot\left(\mathbf{g}_{k} \times \mathbf{g}_{j}\right)\right) \mathbf{g}^{j} \otimes \mathbf{g}^{k}\right) \mathbf{a} & =\sum_{k=1}^{3} \sum_{j=1}^{3}\left(\mathbf{M}_{O} \cdot\left(a^{k} \mathbf{g}_{k} \times \mathbf{g}_{j}\right)\right) \mathbf{g}^{j} \\
& =\sum_{j=1}^{3}\left(\mathbf{M}_{O} \cdot\left(\mathbf{a} \times \mathbf{g}_{j}\right)\right) \mathbf{g}^{j} \\
& =\sum_{j=1}^{3}\left(\left(\mathbf{M}_{O} \times \mathbf{a}\right) \cdot \mathbf{g}_{j}\right) \mathbf{g}^{j} \\
& =\mathbf{M}_{O} \times \mathbf{a} .
\end{aligned}
$$

We conclude that $\mathbf{M}_{O}$ is the axial vector of ${ }_{1} \mathbf{D}-{ }_{1} \mathbf{D}^{T}$. Hence,

$$
{ }_{1} D_{32}-{ }_{1} D_{23}=\mathbf{M}_{O} \cdot \mathbf{E}_{1}, \quad{ }_{1} D_{13}-{ }_{1} D_{31}=\mathbf{M}_{O} \cdot \mathbf{E}_{2}, \quad{ }_{1} D_{21}-{ }_{1} D_{12}=\mathbf{M}_{O} \cdot \mathbf{E}_{3} .
$$

Using (41), the skew-symmetric part of $\mathrm{K}_{O}$ can be shown to have the representation

$$
\frac{1}{2}\left(\mathrm{~K}_{O}-\mathrm{K}_{O}^{T}\right)=\frac{1}{2}\left[\begin{array}{cc}
0 & { }_{1} \mathrm{C} \\
-{ }_{1} \mathrm{C} & \left({ }_{1} \mathrm{D}-{ }_{1} \mathrm{D}^{T}\right)
\end{array}\right],
$$

we conclude that the force $\mathbf{F}$ and moment $\mathbf{M}_{O}$ contribute equally to the skew-symmetric components of $\mathrm{K}_{O}$. 
For the stiffness matrix ${ }_{2} \mathrm{~K}_{c}$, the only matrix which contributes to its skew-symmetric part is ${ }_{2} D_{m n}$. Paralleling the development of (56), we find that

$$
{ }_{2} D_{32}-{ }_{2} D_{23}=\mathbf{M} \cdot \mathbf{E}_{1}, \quad{ }_{2} D_{13}-{ }_{2} D_{31}=\mathbf{M} \cdot \mathbf{E}_{2}, \quad{ }_{2} D_{21}-{ }_{2} D_{12}=\mathbf{M} \cdot \mathbf{E}_{3} .
$$

We emphasize that in contrast to ${ }_{1} \mathrm{~K}_{c}$ and $\mathrm{K}_{O}, \mathbf{F}$ does not contribute to the skew-symmetric part of ${ }_{2} \mathrm{~K}_{c}$. In Sect. 7, examples of the identities (48), (56), and (58) will be shown.

\section{The planar case}

It is of interest to restrict attention to rigid bodies undergoing planar motions in the $\mathbf{E}_{1}-\mathbf{E}_{2}$ plane. An example of such a system is shown in Fig. 3. For the planar case, the sole angle of rotation is $\psi$. Additionally, the dual Euler basis is not needed and the axis of rotation is simply $\mathbf{E}_{3}$. Further,

$$
U=U_{1}\left(\psi, x_{1}, x_{2}\right)=U_{2}\left(\psi, X_{1}, X_{2}\right)=U_{3}\left(\psi, x_{A_{1}}, x_{A_{2}}\right)=U_{4}\left(\psi, X_{A_{1}}, X_{A_{2}}\right) .
$$

It shall shortly become apparent that the Cartesian stiffness matrix ${ }_{2} \mathrm{~K}_{c}$ will be symmetric, while the matrix ${ }_{1} \mathrm{~K}_{c}$ can still retain an asymmetric component provided that the gradient of $U_{1}$ doesn't vanish.

The expression for the stiffness matrix simplifies dramatically in the planar case. First, the stiffness matrix is now defined by the relations

$$
\left[\begin{array}{c}
\left(\mathbf{F}^{\prime}-\mathbf{F}\right) \cdot \mathbf{E}_{1} \\
\left(\mathbf{F}^{\prime}-\mathbf{F}\right) \cdot \mathbf{E}_{2} \\
\left(\mathbf{M}_{O}^{\prime}-\mathbf{M}_{O}\right) \cdot \mathbf{E}_{3}
\end{array}\right]=-{ }_{1} \mathrm{~K}_{c}\left[\begin{array}{c}
\left(\overline{\mathbf{x}}^{\prime}-\overline{\mathbf{x}}\right) \cdot \mathbf{E}_{1} \\
\left(\overline{\mathbf{x}}^{\prime}-\overline{\mathbf{x}}\right) \cdot \mathbf{E}_{2} \\
\psi^{\prime}-\psi
\end{array}\right]
$$

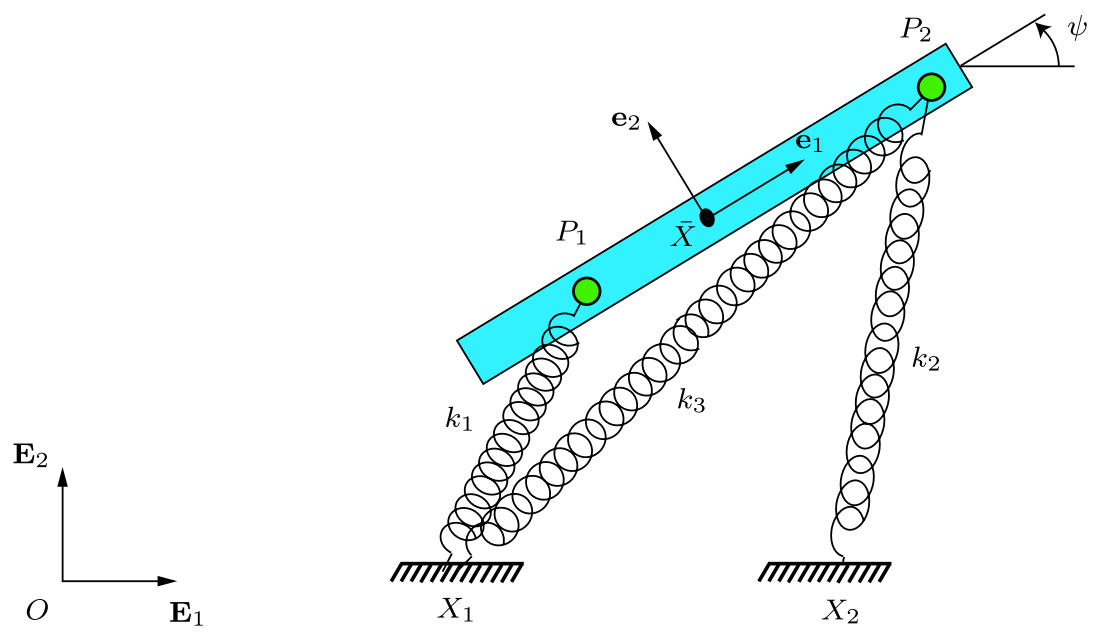

Fig. 3 Schematic of a rigid body which undergoes planar motions. The body is attached to two fixed points $P_{1}$ and $P_{2}$ by springs of stiffnesses $k_{i}$ and unstretched lengths $L_{i}$. This example is identical to one considered by Griffis and Duffy [8] 
Paralleling the developments in Sect. 4.1, we find that the Cartesian stiffness matrix has the representation

$$
\begin{aligned}
{ }_{1} \mathbf{K}_{c}= & \mathbf{Q}^{T}\left[\begin{array}{ccc}
\frac{\partial^{2} U_{1}}{\partial x_{1} \partial x_{1}} & \frac{\partial^{2} U_{1}}{\partial x_{1} \partial x_{2}} & \frac{\partial^{2} U_{1}}{\partial x_{1} \partial \psi} \\
\frac{\partial^{2} U_{1}}{\partial x_{2} \partial x_{1}} & \frac{\partial^{2} U_{1}}{\partial x_{2} \partial x_{2}} & \frac{\partial^{2} U_{1}}{\partial x_{2} \partial \psi} \\
\frac{\partial^{2} U_{1}}{\partial \psi \partial x_{1}} & \frac{\partial^{2} U_{1}}{\partial \psi \partial x_{2}} & \frac{\partial^{2} U_{1}}{\partial \psi \partial \psi}
\end{array}\right] \mathbf{Q}+\left[\begin{array}{ccc}
0 & 0 & \mathbf{F} \cdot \mathbf{E}_{2} \\
0 & 0 & -\mathbf{F} \cdot \mathbf{E}_{1} \\
0 & 0 & 0
\end{array}\right] \\
+ & \mathbf{Q}^{T}\left[\begin{array}{ccc}
\frac{\partial^{2} U_{1}}{\partial x_{1} \partial x_{1}} & \frac{\partial^{2} U_{1}}{\partial x_{1} \partial x_{2}} & 0 \\
\frac{\partial^{2} U_{1}}{\partial x_{2} \partial x_{1}} & \frac{\partial^{2} U_{1}}{\partial x_{2} \partial x_{2}} & 0 \\
\frac{\partial^{2} U_{1}}{\partial \psi \partial x_{1}} & \frac{\partial^{2} U_{1}}{\partial \psi \partial x_{2}} & 0
\end{array}\right]\left[\begin{array}{ccc}
0 & 0 & x_{2} \\
0 & 0 & -x_{1} \\
-x_{2} & x_{1} & 0
\end{array}\right] \mathbf{Q} .
\end{aligned}
$$

Here, the rotation matrix $Q$ is

$$
\mathbf{Q}=\left[\begin{array}{ccc}
\cos (\psi) & \sin (\psi) & 0 \\
-\sin (\psi) & \cos (\psi) & 0 \\
0 & 0 & 1
\end{array}\right]
$$

In writing (61), we choose to express the skew-symmetric components of ${ }_{1} \mathrm{~K}_{c}$ in terms of the force components.

If the force $\mathbf{F}$ and moment $\mathbf{M}$ are used, then we need to repeat the calculation with the function $U_{2}$. In this case, we simply find a symmetric Cartesian stiffness matrix:

$$
\left[\begin{array}{c}
\left(\mathbf{F}^{\prime}-\mathbf{F}\right) \cdot \mathbf{E}_{1} \\
\left(\mathbf{F}^{\prime}-\mathbf{F}\right) \cdot \mathbf{E}_{2} \\
\left(\mathbf{M}^{\prime}-\mathbf{M}\right) \cdot \mathbf{E}_{3}
\end{array}\right]=-\left[\begin{array}{ccc}
\frac{\partial^{2} U_{2}}{\partial X_{1} \partial X_{1}} & \frac{\partial^{2} U_{2}}{\partial X_{1} \partial X_{2}} & \frac{\partial^{2} U_{2}}{\partial X_{1} \partial \psi} \\
\frac{\partial^{2} U_{2}}{\partial X_{2} \partial X_{1}} & \frac{\partial^{2} U_{2}}{\partial X_{2} \partial X_{2}} & \frac{\partial^{2} U_{2}}{\partial X_{2} \partial \psi} \\
\frac{\partial^{2} U_{2}}{\partial \psi \partial X_{1}} & \frac{\partial^{2} U_{2}}{\partial \psi \partial X_{2}} & \frac{\partial^{2} U_{2}}{\partial \psi \partial \psi}
\end{array}\right]\left[\begin{array}{c}
\left(\overline{\mathbf{x}}^{\prime}-\overline{\mathbf{x}}\right) \cdot \mathbf{E}_{1} \\
\left(\overline{\mathbf{x}}^{\prime}-\overline{\mathbf{x}}\right) \cdot \mathbf{E}_{2} \\
\psi^{\prime}-\psi
\end{array}\right] .
$$

The $3 \times 3$ matrix in this equation is a symmetric Cartesian stiffness matrix ${ }_{2} \mathrm{~K}_{c}$. The symmetry of this matrix is independent of the value of the gradient of $U_{2}$.

\section{The Stewart-Gough platform}

To illustrate the previous developments, we turn to the example of the Stewart-Gough platform. As shown in Fig. 4, the realization of this system for the purposes of this paper is that of a rigid platform in the shape of an equilateral triangle which is attached by six springs to a rigid base. The springs define a conservative force field for the platform, and in the sequel we compute its potential energy and Cartesian stiffness matrices. This platform is a featured example in several other works on the Cartesian stiffness matrix [5, 8, 21]. For the purpose of comparison, we consider the same parameter values as these works.

\subsection{Preliminary kinematic considerations}

The springs have stiffnesses of $k_{1}, \ldots k_{6}$ and unstretched lengths of $l_{01}, \ldots, l_{06}$, respectively. We follow $[5,8,21]$ and specify the parameter values

$$
l_{01}=11, \quad l_{02}=12, \quad l_{03}=13, \quad l_{04}=14, \quad l_{05}=15, \quad l_{06}=16,
$$




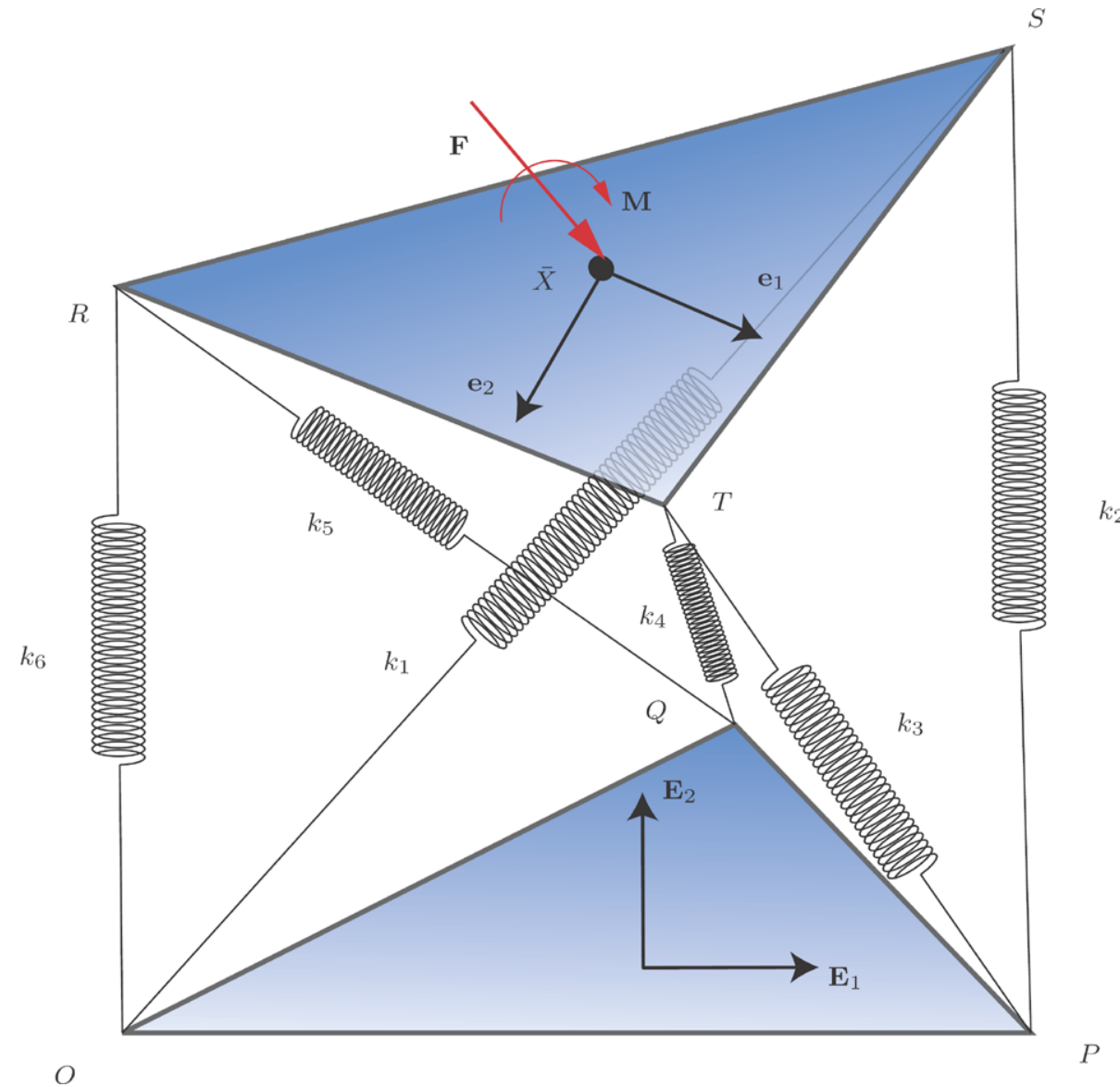

Fig. 4 Schematic of a realization of a six degree-of-freedom system known as a Stewart-Gough platform. Here, a rigid body which undergoes planar motions is attached to three fixed points $O, P, Q$ by linear springs. This example is identical to one considered by Griffis and Duffy [8] and the related publications [5, 21]

$$
k_{1}=10, \quad k_{2}=20, \quad k_{3}=30, \quad k_{4}=40, \quad k_{5}=50, \quad k_{6}=60 .
$$

The lengths are prescribed in centimeters and the stiffnesses are prescribed in $\mathrm{N} / \mathrm{cm}$. The position vectors of the two points $P$ and $Q$ are

$$
\mathbf{r}_{P}=7 \mathbf{E}_{1}, \quad \mathbf{r}_{Q}=3.5 \mathbf{E}_{1}+3.5 \sqrt{3} \mathbf{E}_{2}
$$

The configuration $\boldsymbol{\kappa}_{t}$ of the platform is defined by the position vector $\overline{\mathbf{x}}$ of the center of mass and the set of 3-2-1 Euler angles values:

$$
\begin{gathered}
\overline{\mathbf{x}}=12.8457 \mathbf{E}_{1}+4.3709 \mathbf{E}_{2}+14.8457 \mathbf{E}_{3}, \\
v^{1}=-33.0826^{\circ}, \quad v^{2}=-39.9638^{\circ}, \quad v^{3}=202.701^{\circ} .
\end{gathered}
$$


For the configuration $\kappa_{t}$ of interest, the position vectors of the points $R, S$, and $T$ are

$$
\mathbf{x}_{R}=\overline{\mathbf{x}}-3.5 \mathbf{e}_{1}+\frac{3.5}{\sqrt{3}} \mathbf{e}_{2}, \quad \mathbf{x}_{S}=\overline{\mathbf{x}}-\frac{7}{\sqrt{3}} \mathbf{e}_{2}, \quad \mathbf{x}_{T}=\overline{\mathbf{x}}+3.5 \mathbf{e}_{1}+\frac{3.5}{\sqrt{3}} \mathbf{e}_{2} .
$$

The reader is referred to Fig. 4 for an illustration of some of these vectors. Representations for the corotational basis vectors in the configuration $\boldsymbol{\kappa}_{t}$ are obtained using (124):

$$
\begin{aligned}
& \mathbf{e}_{1}=0.642198 \mathbf{E}_{1}-0.41832 \mathbf{E}_{2}+0.642332 \mathbf{E}_{3}, \\
& \mathbf{e}_{2}=-0.29577 \mathbf{E}_{1}-0.9083 \mathbf{E}_{2}-0.295824 \mathbf{E}_{3}, \\
& \mathbf{e}_{3}=0.707179 \mathbf{E}_{1}-0.707035 \mathbf{E}_{3} .
\end{aligned}
$$

With the help of (126), representations for the dual Euler basis vectors can be found:

$$
\begin{aligned}
& \mathbf{g}^{1}=-0.702167 \mathbf{E}_{1}+0.457433 \mathbf{E}_{2}+\mathbf{E}_{3}, \\
& \mathbf{g}^{2}=0.545847 \mathbf{E}_{1}+0.837885 \mathbf{E}_{2}, \\
& \mathbf{g}^{3}=1.0932 \mathbf{E}_{1}-0.712176 \mathbf{E}_{2} .
\end{aligned}
$$

The potential energy function for the platform can be obtained by adding the potential energies of each of the springs:

$$
V=\sum_{J=1}^{6} \frac{k_{J}}{2}\left(l_{J}-l_{0 J}\right)^{2},
$$

where $l_{1}, \ldots, l_{6}$ are the stretched lengths of the springs. The configuration $\kappa_{t}$ is held in equilibrium by a force $\mathbf{F}$ acting at the center of mass and a moment $\mathbf{M}$ relative to the center of mass. These quantities are obtained using the potential energy function $V$ and the representations (16):

$$
\begin{aligned}
\mathbf{F}=-\sum_{k=1}^{3} \frac{\partial V_{2}}{\partial X_{k}} \mathbf{E}_{k} & =-304.649 \mathbf{E}_{1}-59.3016 \mathbf{E}_{2}-505.968 \mathbf{E}_{3}, \\
\mathbf{M =}=-\sum_{k=1}^{3} \frac{\partial V_{2}}{\partial \nu^{k}} \mathbf{g}^{k} & =-200.324 \mathbf{g}^{1}+545.558 \mathbf{g}^{2}-47.945 \mathbf{g}^{3} \\
& =386.039 \mathbf{E}_{1}+399.625 \mathbf{E}_{2}-200.324 \mathbf{E}_{3}
\end{aligned}
$$

The system (71) is equipollent to a force $\mathbf{F}$ and a moment $\mathbf{M}_{O}$ where

$$
\begin{aligned}
\mathbf{F}=-\sum_{k=1}^{3} \frac{\partial V_{1}}{\partial x_{k}} \mathbf{e}_{k} & =-495.82 \mathbf{e}_{1}+293.658 \mathbf{e}_{2}+142.333 \mathbf{e}_{3} \\
& =-304.649 \mathbf{E}_{1}-59.3016 \mathbf{E}_{2}-505.968 \mathbf{E}_{3}, \\
\mathbf{M}_{O}=-\sum_{k=1}^{3} \frac{\partial V_{1}}{\partial \nu^{k}} \mathbf{g}^{k} & =369.493 \mathbf{g}^{1}+1475.27 \mathbf{g}^{2}-1363.84 \mathbf{g}^{3} \\
& =-945.122 \mathbf{E}_{1}+2376.42 \mathbf{E}_{2}+369.493 \mathbf{E}_{3} .
\end{aligned}
$$




\subsection{Stiffness matrices}

It is straightforward to compute the stiffness matrices associated with the potential energy $V$ for the configuration $\kappa_{t}$. With the help of (28) and (41), we find that

$$
\begin{aligned}
& \mathrm{K}_{O}=\left[\begin{array}{rrrrrr}
80.0014 & 5.20958 & 75.5599 & 206.947 & -202.424 & -180.249 \\
5.20958 & 39.3191 & 5.20994 & -75.5072 & 5.269 & 212.017 \\
75.5599 & 5.20994 & 150.613 & 407.245 & -532.151 & -212.216 \\
206.947 & -75.5072 & 407.245 & 4779.91 & -1693.32 & -3895.24 \\
-202.424 & 5.269 & -532.151 & -1693.32 & 2705.38 & -336.504 \\
-180.249 & 212.017 & -212.216 & -3895.24 & -336.504 & 3264.02
\end{array}\right] \\
& +\left[\begin{array}{rrrrrr}
0 & 0 & 0 & 0 & 505.968 & -59.3016 \\
0 & 0 & 0 & -505.968 & 0 & 304.649 \\
0 & 0 & 0 & 59.3016 & -304.649 & 0 \\
0 & 0 & 0 & -2638.32 & -401.422 & 2376.42 \\
0 & 0 & 0 & -31.9282 & 1402.01 & 945.122 \\
0 & 0 & 0 & 0 & 0 & 0
\end{array}\right] \\
& { }_{1} \mathrm{~K}_{c}=\mathrm{K}_{O}+\left[\begin{array}{rrrrrr}
0 & 0 & 0 & -252.925 & -217.057 & 282.757 \\
0 & 0 & 0 & 560.947 & -10.4145 & -482.311 \\
0 & 0 & 0 & -580.968 & 812.987 & 263.339 \\
0 & 0 & 0 & -2900.98 & 2159.07 & 1874.49 \\
0 & 0 & 0 & 2404.2 & -3830.72 & -952.461 \\
0 & 0 & 0 & 4075.12 & -50.1405 & -3511.36
\end{array}\right]
\end{aligned}
$$

It is interesting to note that the Hessian of $V_{1}$ has the following value:

$$
\left[\begin{array}{ll}
{ }_{1} \mathrm{~K}_{1} & { }_{1} \mathrm{~K}_{3} \\
{ }_{1} \mathrm{~K}_{3}^{T} & { }_{1} \mathrm{~K}_{2}
\end{array}\right]=\left[\begin{array}{rrrrrr}
158.747 & -62.3749 & -32.0617 & -340.763 & -164.594 & 253.051 \\
-62.3749 & 71.4421 & 14.7679 & -76.4696 & 116.32 & -210.66 \\
-32.0617 & 14.7679 & 39.744 & 22.5743 & 118.039 & -173.972 \\
-340.763 & -76.4696 & 22.5743 & 3264.02 & -2408.16 & -264.238 \\
-164.594 & 116.32 & 118.039 & -2408.16 & 1774.58 & -1344.02 \\
253.051 & -210.66 & -173.972 & -264.238 & -1344.02 & 1668.71
\end{array}\right] .
$$

This Hessian has a spectrum

$$
\left[\lambda_{1}, \ldots, \lambda_{6}\right]=[-711.826,20.9161,49.4755,117.917,2343.64,5157.12] .
$$

Due to the nonzero values of $\mathbf{F}$ and $\mathbf{M}_{O}$, the matrix $\mathrm{K}_{O}$ is asymmetric. We also observe that the components of the skew-symmetric matrix ${ }_{1} \mathrm{C}$ and the matrix ${ }_{1} \mathrm{D}$ which feature in (73) satisfy the identities (48) and (56). The value for $\mathrm{K}_{O}$ in (73) is identical to the expression for the stiffness matrix $\hat{\mathbf{K}}_{O}$ recorded in (50) of Ciblak and Lipkin [5] although their methods are different to ours. 
The stiffness matrix ${ }_{2} \mathrm{~K}_{c}$ is distinct from ${ }_{1} \mathrm{~K}_{c}$. With the help of (35), it is straightforward to compute that

$$
\begin{aligned}
{ }_{2} \mathrm{~K}_{c}= & {\left[\begin{array}{rrrrrr}
80.0014 & 5.20958 & 75.5599 & -45.9777 & 86.4865 & 43.2066 \\
5.20958 & 39.3191 & 5.20994 & -20.5279 & -5.14554 & 34.355 \\
75.5599 & 5.20994 & 150.613 & -114.422 & -23.812 & 51.1232 \\
-45.9777 & -20.5279 & -114.422 & -415.399 & -293.389 & 242.607 \\
86.4865 & -5.14554 & -23.812 & -293.389 & -1004.59 & 57.4772 \\
43.2066 & 34.355 & 51.1232 & 242.607 & 57.4772 & -499.799
\end{array}\right] } \\
& +\left[\begin{array}{rrrrrr}
0 & 0 & 0 & 0 & 0 & 0 \\
0 & 0 & 0 & 0 & 0 & 0 \\
0 & 0 & 0 & 0 & 0 & 0 \\
0 & 0 & 0 & -148.617 & 385.404 & 399.625 \\
0 & 0 & 0 & 185.08 & -308.574 & -386.039 \\
0 & 0 & 0 & 0 & 0 & 0
\end{array}\right] .
\end{aligned}
$$

Clearly, this matrix is asymmetric. We also observe that the components of the skewsymmetric part of the matrix ${ }_{2} \mathrm{D}$ which feature in (76) satisfy the identities (58). In addition, the values of the matrices ${ }_{1} \mathrm{~K}_{c}$ and ${ }_{2} \mathrm{~K}_{c}$ in (73) and (76) satisfy the identities (39).

It is interesting to note that one of the constituents of ${ }_{2} \mathrm{~K}_{c}$, the Hessian of $V_{2}$, has the value

$$
\left[\begin{array}{ll}
{ }_{2} \mathrm{~K}_{1} & { }_{2} \mathrm{~K}_{3} \\
{ }_{2} \mathrm{~K}_{3}^{T} & { }_{2} \mathrm{~K}_{2}
\end{array}\right]=\left[\begin{array}{rrrrrr}
80.0014 & 5.20958 & 75.5599 & 43.2066 & 47.3689 & -37.9579 \\
5.20958 & 39.3191 & 5.20994 & 34.355 & -15.5164 & 11.0361 \\
75.5599 & 5.20994 & 150.613 & 51.1232 & -82.4086 & -30.6826 \\
43.2066 & 34.355 & 51.1232 & -499.799 & 180.586 & -189.268 \\
47.3689 & -15.5164 & -82.4086 & 180.586 & -1097.41 & 231.658 \\
-37.9579 & 11.0361 & -30.6826 & -189.268 & 231.658 & -226.44
\end{array}\right] .
$$

The Hessian of $V_{2}$ has a spectrum

$$
\left[\mu_{1}, \ldots, \mu_{6}\right]=[-1231.46,-492.751,-128.017,38.0753,41.9597,218.474] .
$$

As the spectra of the Hessians of $V_{1}$ and $V_{2}$ are distinct (cf. (75) and (78)) they cannot be related by a similarity transformation.

\section{Multibody systems}

Suppose now that our system is composed of two rigid bodies with positions of the center of mass and rotation tensor associated with the $K$ th body denoted by $\overline{\mathbf{x}}^{K}$ and $\mathbf{Q}_{K}$ respectively. That is, the corotational basis vectors fixed to body $K$ is given by $\mathbf{e}_{i}^{K}=\mathbf{Q}_{K} \mathbf{E}_{i}$ (cf. Fig. 5). Echoing the development in Sect. 2, we have the representations,

$$
\begin{aligned}
& \overline{\mathbf{x}}^{K}=X_{1}^{K} \mathbf{E}_{1}+X_{2}^{K} \mathbf{E}_{2}+X_{3}^{K} \mathbf{E}_{3}=x_{1}^{K} \mathbf{e}_{1}^{K}+x_{2}^{K} \mathbf{e}_{2}^{K}+x_{3}^{K} \mathbf{e}_{3}^{K}, \\
& \mathbf{x}_{A}^{K}=X_{A_{1}}^{K} \mathbf{E}_{1}+X_{A_{2}}^{K} \mathbf{E}_{3}+X_{A_{3}}^{K} \mathbf{E}_{3}=x_{A_{1}}^{K} \mathbf{e}_{1}^{K}+x_{A_{2}}^{K} \mathbf{e}_{2}^{K}+x_{A_{3}}^{K} \mathbf{e}_{3}^{K} \quad(K=1,2) .
\end{aligned}
$$

Unless specified, we use capital letters when the components of the vector on the $K$ th body are written in terms of the fixed basis and lowercase letters when they are written in terms of the basis vectors fixed to the $K$ th body. 
Fig. 5 An example of a rigid body system composed of two bodies. The functional spinal unit shown consists of the sacrum $\mathcal{S}$, the fifth lumbar vertebra $\mathcal{L}_{5}$, and the intervertebral disc $\mathcal{I}$. The basis vectors $\left\{\mathbf{e}_{1}^{1}, \mathbf{e}_{3}^{1}, \mathbf{e}_{3}^{1}\right\}$ and $\left\{\mathbf{e}_{1}^{2}, \mathbf{e}_{3}^{2}, \mathbf{e}_{3}^{2}\right\}$ are attached to the body $\mathcal{S}$ and $\mathcal{L}_{5}$ respectively. The conservative forces $\left(\mathbf{F}_{1}\right.$ and $\left.\mathbf{F}_{2}\right)$ and moments $\left(\mathbf{M}_{1}\right.$ and $\left.\mathbf{M}_{2}\right)$ supplied by the disc, facets, and ligaments to the two vertebral units are given by (88) and (95)-(94)

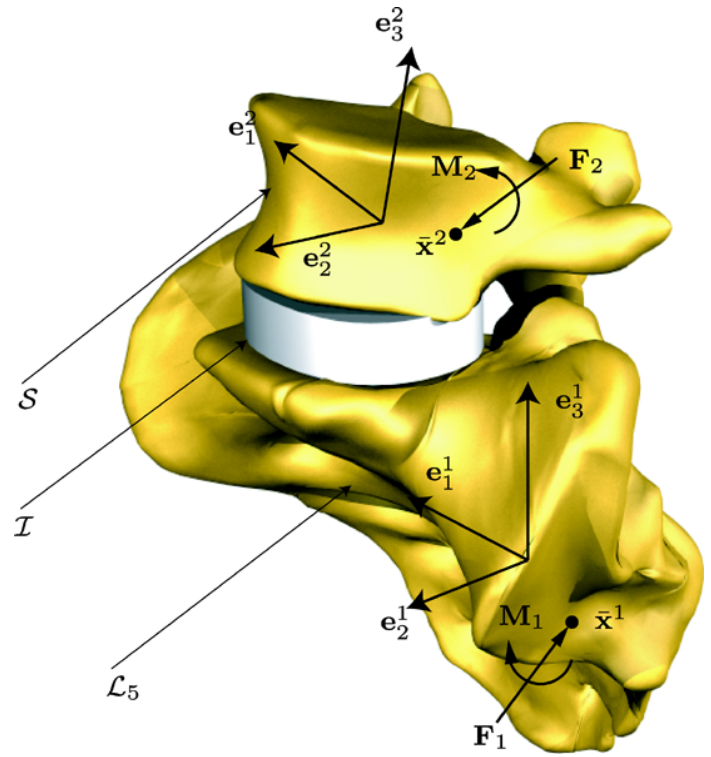

We denote the Euler angles used to parameterize the rotation tensor $\mathbf{Q}_{K}$ of the $K$ th rigid body by $\left(v_{K}^{1}, v_{K}^{2}, v_{K}^{3}\right)$. Likewise, $\left(\beta^{1}, \beta^{2}, \beta^{3}\right)$ are the three Euler angles used to characterize the relative rotation between the two bodies:

$$
\begin{aligned}
& \mathbf{Q}_{1}=\mathbf{Q}_{1}\left(v_{1}^{1}, v_{1}^{2}, v_{1}^{3}\right), \\
& \mathbf{Q}_{2}=\mathbf{Q}_{2}\left(v_{2}^{1}, v_{2}^{2}, v_{2}^{3}\right), \\
& \mathbf{R}=\mathbf{Q}_{2}\left(\mathbf{Q}_{1}\right)^{T}=\mathbf{R}\left(\beta^{1}, \beta^{2}, \beta^{3}\right) .
\end{aligned}
$$

It follows that the angular velocity vectors of the first and second rigid bodies have the representations

$$
\begin{aligned}
\boldsymbol{\omega}_{1} & =\dot{v}_{1}^{1} \mathbf{g}_{1}^{1}+\dot{v}_{1}^{2} \mathbf{g}_{2}^{1}+\dot{v}_{1}^{3} \mathbf{g}_{3}^{1}, \\
\boldsymbol{\omega}_{2} & =\dot{v}_{2}^{1} \mathbf{g}_{1}^{2}+\dot{v}_{2}^{2} \mathbf{g}_{2}^{2}+\dot{v}_{2}^{3} \mathbf{g}_{3}^{2} \\
& =\boldsymbol{\omega}_{1}+\boldsymbol{\omega}_{\text {rel }} \\
& =\left(\dot{v}_{1}^{1} \mathbf{g}_{1}^{1}+\dot{v}_{1}^{2} \mathbf{g}_{2}^{1}+\dot{v}_{1}^{3} \mathbf{g}_{3}^{1}\right)+\left(\dot{\beta}^{1} \mathbf{g}_{1}^{\text {rel }}+\dot{\beta}^{2} \mathbf{g}_{2}^{\text {rel }}+\dot{\beta}^{1} \mathbf{g}_{3}^{\text {rel }}\right) .
\end{aligned}
$$

For the $K$ th rigid body, $\left\{\mathbf{g}_{1}^{K}, \mathbf{g}_{2}^{K}, \mathbf{g}_{3}^{K}\right\}$ are the Euler basis vectors with a dual basis denoted by $\left\{\mathbf{g}^{K, 1}, \mathbf{g}^{K, 2}, \mathbf{g}^{K, 3}\right\}$. Further, $\left\{\mathbf{g}_{1}^{\text {rel }}, \mathbf{g}_{2}^{\text {rel }}, \mathbf{g}_{3}^{\text {rel }}\right\}$ is the Euler basis of the relative rotation between the two bodies with a dual basis denoted by $\left\{\mathbf{g}^{\text {rel, } 1}, \mathbf{g}^{\text {rel, }, 2}, \mathbf{g}^{\text {rel, } 3}\right\} .^{2}$

\subsection{Potential energy functions}

Depending on the system of interest, several different representations of the potential energy function for a system of two rigid bodies are possible. To elaborate, consider the three

\footnotetext{
${ }^{2}$ It is important to note that the Euler angles are not additive: $v_{2}^{i} \neq v_{1}^{i}+\beta^{i}(i=1,2,3)$.
} 


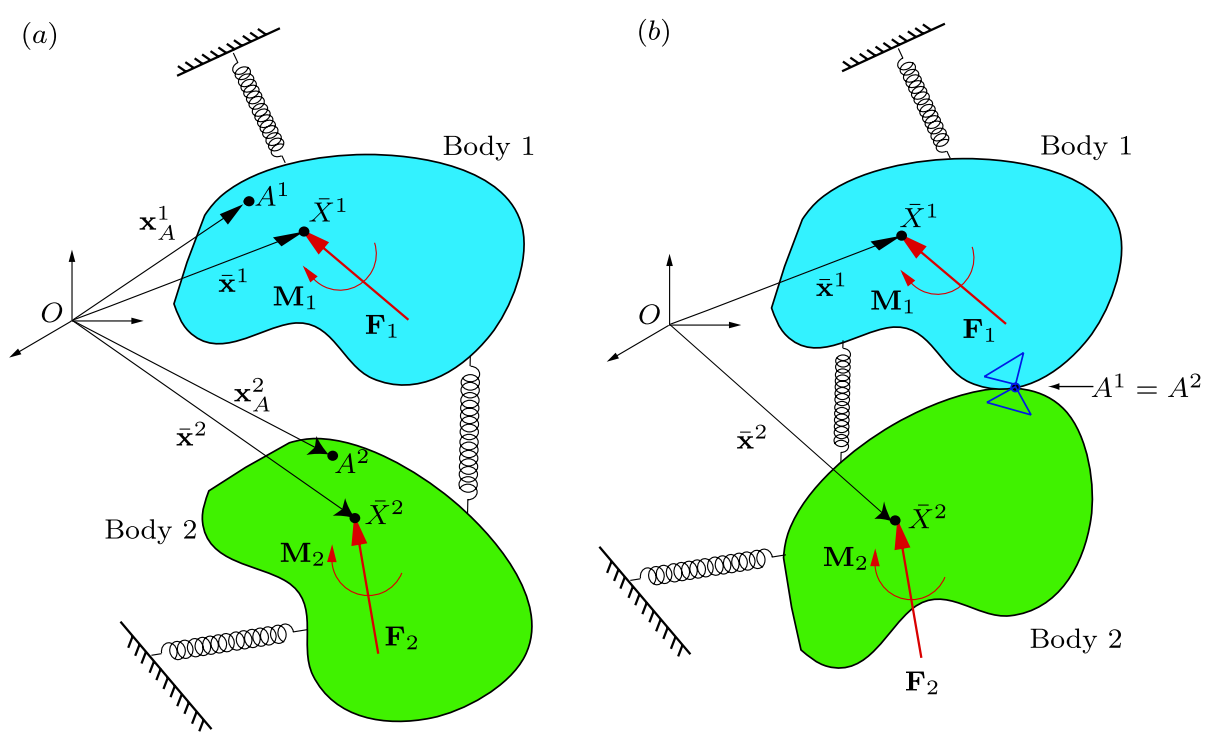

(c)

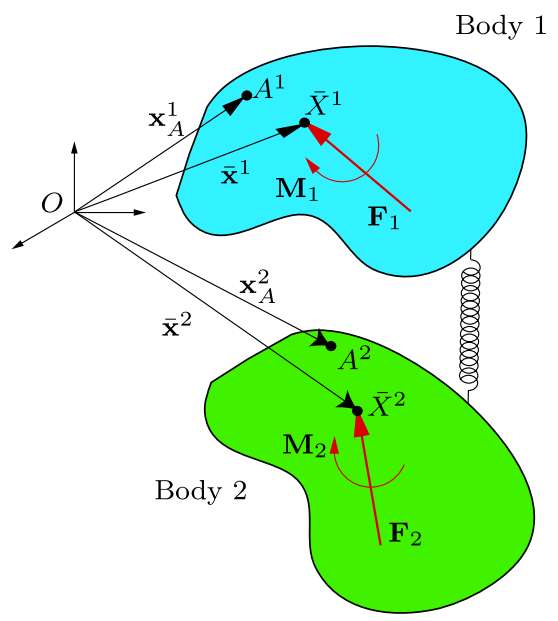

Fig. 6 Three different systems of rigid bodies: In (a), the bodies are connected by springs to each other and a fixed surface, in (b), the bodies are also pin jointed at $A$, and, in (c), the bodies are connected to each other by springs and are otherwise isolated from the environment

examples shown in Fig. 6. In the first example, shown in Fig. 6(a), each of the bodies are connected to the ground by springs and also connected to each other by elastic springs. The potential energy of this system will depend on the absolute motion of the centers of mass of the bodies and the rotation tensor of each body. As a modification to this case, suppose that the bodies are now connected by a joint at a point $A$ (see Fig. 6(b)). For this case, $U$ can be expressed as a function of the position vector of $A$ and the rotation tensor of each body. Finally, when the only conservative forces and moments acting on the pair of rigid bodies are due to their interactions with each other, then $U$ can be expressed as a function of the relative position vector of their centers of mass and the relative rotation tensor $\mathbf{R}$ of 
the bodies. As emphasized in $[14,15]$, this situation arises in celestial mechanics problems where the two bodies are attracted to each other by a central force field and in problems featuring pairs of rigid bodies attached by elastic springs (see Fig. 6(c)).

When applicable, the easiest representation of $U$ to work with arises when this function depends only on the relative position vector $\mathbf{y}$ and relative rotation tensor $\mathbf{R}$ :

$$
\overline{\mathbf{y}}=\overline{\mathbf{x}}^{2}-\overline{\mathbf{x}}^{1}=\sum_{i=1}^{3} Y_{i} \mathbf{E}_{i}, \quad \mathbf{R}=\mathbf{Q}_{2} \mathbf{Q}_{1}^{T}=\mathbf{R}\left(\beta^{1}, \beta^{2}, \beta^{3}\right) .
$$

In the more general case, the potential energy function depends on position vectors of points on each body and the rotation tensors of each body. Following our discussion of the situations shown in Fig. 6, we need to consider several distinct representations for $U$ :

$$
\begin{aligned}
& U=\hat{U}_{1}\left(\mathbf{Q}_{1}, \mathbf{Q}_{2}, \overline{\mathbf{x}}^{1}, \overline{\mathbf{x}}^{2}\right), \\
& U=\hat{U}_{2}\left(\mathbf{Q}_{1}, \mathbf{Q}_{2}, \overline{\mathbf{x}}^{1}, \overline{\mathbf{x}}^{2}\right), \\
& U=\hat{U}_{3}\left(\mathbf{Q}_{1}, \mathbf{R}, \mathbf{y}\right), \\
& U=\hat{U}_{4}\left(\mathbf{Q}_{1}, \mathbf{Q}_{2}, \mathbf{x}_{A}^{1}, \mathbf{x}_{A}^{2}\right), \\
& U=\hat{U}_{5}(\mathbf{R}, \mathbf{y}), \\
& U=\hat{U}_{6}\left(\mathbf{Q}_{1}, \mathbf{R}, \mathbf{x}_{A}^{1}, \mathbf{x}_{A}^{2}\right) .
\end{aligned}
$$

These representations have the respective component forms,

$$
\begin{aligned}
& U=U_{1}\left(v_{1}^{1}, v_{1}^{2}, v_{1}^{3}, v_{2}^{1}, v_{2}^{2}, v_{2}^{3}, x_{1}^{1}, x_{2}^{1}, x_{3}^{1}, x_{1}^{2}, x_{2}^{2}, x_{3}^{2}\right), \\
& U=U_{2}\left(v_{1}^{1}, v_{1}^{2}, v_{1}^{3}, v_{2}^{1}, v_{2}^{2}, v_{2}^{3}, X_{1}^{1}, X_{2}^{1}, X_{3}^{1}, X_{1}^{2}, X_{2}^{2}, X_{3}^{2}\right), \\
& U=U_{3}\left(v_{1}^{1}, v_{1}^{2}, v_{1}^{3}, \beta^{1}, \beta^{2}, \beta^{3}, Y_{1}, Y_{2}, Y_{3}\right), \\
& U=U_{4}\left(v_{1}^{1}, v_{1}^{2}, v_{1}^{3}, v_{2}^{1}, v_{2}^{2}, v_{2}^{3}, X_{A_{1}}^{1}, X_{A_{2}}^{1}, X_{A_{3}}^{1}, X_{A_{1}}^{2}, X_{A_{2}}^{2}, X_{A_{3}}^{2}\right), \\
& U=U_{5}\left(\beta^{1}, \beta^{2}, \beta^{3}, Y_{1}, Y_{2}, Y_{3}\right), \\
& U=U_{6}\left(v_{1}^{1}, v_{1}^{2}, v_{1}^{3}, \beta^{1}, \beta^{2}, \beta^{3}, X_{A_{1}}^{1}, X_{A_{2}}^{1}, X_{A_{3}}^{1}, X_{A_{1}}^{2}, X_{A_{2}}^{2}, X_{A_{3}}^{2}\right) .
\end{aligned}
$$

In certain cases, the potential energy function $U$ can also be written as functions of the relative position vector $\mathbf{y}$, or points $\mathbf{x}_{A}^{1}$ and $\mathbf{x}_{A}^{2}$ on the two bodies, all expressed in terms of their components in the bases vectors fixed to the respective bodies. However, these representations cannot be used to derive the simple representations given below in (88) and (95)-(94) of the conservative forces and moments.

\subsection{The case $U=\hat{U}_{2}\left(\mathbf{Q}_{1}, \mathbf{Q}_{2}, \overline{\mathbf{x}}^{1}, \overline{\mathbf{x}}^{2}\right)$}

The expression for the potential function as

$$
U=U_{2}\left(v_{1}^{1}, v_{1}^{2}, v_{1}^{3}, v_{2}^{1}, v_{2}^{2}, v_{2}^{3}, X_{1}^{1}, X_{2}^{1}, X_{3}^{1}, X_{1}^{2}, X_{2}^{2}, X_{3}^{2}\right)
$$

is a direct extension of the potential $U_{2}$ presented for the single rigid body case. To determine the conservative forces, $\mathbf{F}_{1}$ and $\mathbf{F}_{2}$, and conservative moments, $\mathbf{M}_{1}$ and $\mathbf{M}_{2}$, taken relative to 
the center of mass of the individual bodies, we once again follow [15] and write

$$
-\dot{U}=\mathbf{F}_{1} \cdot \dot{\overline{\mathbf{x}}}^{1}+\mathbf{F}_{2} \cdot \dot{\overline{\mathbf{x}}}^{2}+\mathbf{M}_{1} \cdot \boldsymbol{\omega}_{1}+\mathbf{M}_{2} \cdot \boldsymbol{\omega}_{2}
$$

Expressing $\dot{U}$ as,

$$
\dot{U}=\sum_{i=1}^{3}\left(\frac{\partial U_{2}}{\partial X_{i}^{1}} \dot{X}_{i}^{1}+\frac{\partial U_{2}}{\partial X_{i}^{2}} \dot{X}_{i}^{2}+\frac{\partial U_{2}}{\partial v_{1}^{i}} \dot{v}_{1}^{i}+\frac{\partial U_{2}}{\partial v_{2}^{i}} \dot{v}_{2}^{i}\right),
$$

it can be concluded that

$$
\mathbf{F}_{1}=-\sum_{k=1}^{3} \frac{\partial U_{2}}{\partial X_{k}^{1}} \mathbf{E}_{k}, \quad \mathbf{F}_{2}=-\sum_{k=1}^{3} \frac{\partial U_{2}}{\partial X_{k}^{2}} \mathbf{E}_{k},
$$

and

$$
\mathbf{M}_{1}=-\sum_{k=1}^{3} \frac{\partial U_{2}}{\partial v_{1}^{k}} \mathbf{g}^{1, k}, \quad \mathbf{M}_{2}=-\sum_{k=1}^{3} \frac{\partial U_{2}}{\partial v_{2}^{k}} \mathbf{g}^{2, k} .
$$

We emphasize that the force $\mathbf{F}_{K}$ in this expression is assumed to act at the center of mass $X^{K}$ and the moment $\mathbf{M}_{K}$ is taken relative to the center of mass (cf. Figs. 5 and 6).

\subsection{The case $U=\hat{U}_{3}\left(\mathbf{Q}_{1}, \mathbf{R}, \mathbf{y}\right)$}

For the case where $U=\hat{U}_{3}\left(\mathbf{Q}_{1}, \mathbf{R}, \mathbf{y}\right)$, we again start with the assumption (86). Using the identities $\omega_{2}=\omega_{1}+\omega_{\text {rel }}$ and $\dot{\mathbf{y}}=\dot{\overline{\mathbf{x}}}_{2}-\dot{\overline{\mathbf{x}}}_{1}$, it can be concluded that

$$
\begin{aligned}
\mathbf{F}_{2}=-\mathbf{F}_{1} & =-\sum_{k=1}^{3} \frac{\partial U_{3}}{\partial Y_{k}} \mathbf{E}_{k}, \\
\mathbf{M}_{1} & =-\sum_{k=1}^{3} \frac{\partial U_{3}}{\partial v_{1}^{k}} \mathbf{g}^{1, k}+\sum_{k=1}^{3} \frac{\partial U_{3}}{\partial \beta^{k}} \mathbf{g}^{\mathrm{rel}, k}, \\
\mathbf{M}_{2} & =-\sum_{k=1}^{3} \frac{\partial U_{3}}{\partial \beta^{k}} \mathbf{g}^{\mathrm{rel}, k}
\end{aligned}
$$

The Cartesian stiffness matrix in this case will be a $12 \times 9$ matrix which relates the 12 Cartesian components $\mathbf{F}_{1} \cdot \mathbf{E}_{k}, \mathbf{F}_{2} \cdot \mathbf{E}_{k}, \mathbf{M}_{1} \cdot \mathbf{E}_{k}$, and $\mathbf{M}_{2} \cdot \mathbf{E}_{k}$ to the 3 Cartesian components of $\mathbf{y}$, and the components of the axial vectors of $\Delta \mathbf{Q}_{1}$ and $\Delta \mathbf{R}$. The details on the derivation of this matrix is similar to those featuring in the development of (35) in Sect. 4.2.

8.4 The cases $U=\hat{U}_{1}\left(\mathbf{Q}_{1}, \mathbf{Q}_{2}, \overline{\mathbf{x}}^{1}, \overline{\mathbf{x}}^{2}\right)$ and $U=\hat{U}_{4}\left(\mathbf{Q}_{1}, \mathbf{Q}_{2}, \overline{\mathbf{x}}_{A}^{1}, \overline{\mathbf{x}}_{A}^{2}\right)$

If we wish to consider moments relative to the points $\mathbf{x}_{A}^{1}$ and $\mathbf{x}_{A}^{2}$, or the origin $O,(12)$ and (13) of Sect. 3 can be used to show that

$$
\begin{aligned}
\mathbf{F}_{1} \cdot \dot{\overline{\mathbf{x}}}^{1}+\mathbf{F}_{2} \cdot \dot{\overline{\mathbf{x}}}^{2}+\mathbf{M}_{1} \cdot \omega_{1}+\mathbf{M}_{2} \cdot \boldsymbol{\omega}_{2}= & \mathbf{F}_{1} \cdot \dot{\mathbf{x}}_{A}^{1}+\mathbf{F}_{2} \cdot \dot{\mathbf{x}}_{A}^{2}+\mathbf{M}_{1, A} \cdot \boldsymbol{\omega}_{1}+\mathbf{M}_{2, A} \cdot \boldsymbol{\omega}_{2} \\
= & \mathbf{F}_{1} \cdot\left(\dot{\mathbf{x}}^{1}-\omega_{1} \times \overline{\mathbf{x}}^{1}\right)+\mathbf{F}_{2} \cdot\left(\dot{\mathbf{x}}^{2}-\omega_{2} \times \overline{\mathbf{x}}^{2}\right) \\
& +\mathbf{M}_{1, O} \cdot \omega_{1}+\mathbf{M}_{2, O} \cdot \omega_{2} .
\end{aligned}
$$


It is helpful to note that the corotational derivatives in (91) are given in terms of basis vectors fixed to the respective bodies. For instance,

$$
\dot{\overline{\mathbf{x}}}^{1}-\boldsymbol{\omega}_{1} \times \overline{\mathbf{x}}^{1}=\sum_{k=1}^{3} \dot{x}_{k}^{1} \mathbf{e}_{k}^{1}, \quad \dot{\overline{\mathbf{x}}}^{2}-\boldsymbol{\omega}_{2} \times \overline{\mathbf{x}}^{2}=\sum_{k=1}^{3} \dot{x}_{k}^{2} \mathbf{e}_{k}^{2} .
$$

Thus, invoking (86), we can establish the identities

$$
\begin{array}{cc}
\mathbf{F}_{1}=-\sum_{k=1}^{3} \frac{\partial U_{1}}{\partial x_{k}^{1}} \mathbf{e}_{k}^{1}, & \mathbf{F}_{2}=-\sum_{k=1}^{3} \frac{\partial U_{1}}{\partial x_{k}^{2}} \mathbf{e}_{k}^{2}, \\
\mathbf{M}_{1, O}=-\sum_{k=1}^{3} \frac{\partial U_{1}}{\partial v_{1}^{k}} \mathbf{g}^{1, k}, & \mathbf{M}_{2, O}=-\sum_{k=1}^{3} \frac{\partial U_{1}}{\partial \nu_{2}^{k}} \mathbf{g}^{2, k},
\end{array}
$$

and

$$
\begin{array}{cc}
\mathbf{F}_{1}=-\sum_{k=1}^{3} \frac{\partial U_{4}}{\partial X_{A_{k}}^{1}} \mathbf{E}_{k}, \quad \mathbf{F}_{2}=-\sum_{k=1}^{3} \frac{\partial U_{4}}{\partial X_{A_{k}}^{2}} \mathbf{E}_{k}, \\
\mathbf{M}_{1, A}=-\sum_{k=1}^{3} \frac{\partial U_{4}}{\partial \nu_{1}^{k}} \mathbf{g}^{1, k}, \quad \mathbf{M}_{2, A}=-\sum_{k=1}^{3} \frac{\partial U_{4}}{\partial \nu_{2}^{k}} \mathbf{g}^{2, k} .
\end{array}
$$

In contrast to (88), (93) illustrates, once again, how the natural representation for the conservative force associated with the rigid body of interest is with respect to the corotational basis of that body when moments about a fixed point $O$ are considered. This is a direct consequence of (92).

The development of the Cartesian stiffness matrices for the functions $U_{1}$ and $U_{4}$ will be similar to the derivation of ${ }_{1} \mathrm{~K}_{c}$ (see (28)). However, the resulting matrix for $U_{1}$ will be a $12 \times 12$ matrix which will relate the Cartesian components $\mathbf{F}_{1} \cdot \mathbf{E}_{k}, \mathbf{F}_{2} \cdot \mathbf{E}_{k}, \mathbf{M}_{1, O} \cdot \mathbf{E}_{k}$, and $\mathbf{M}_{2, O} \cdot \mathbf{E}_{k}$ to the Cartesian components of $\overline{\mathbf{x}}_{1}, \overline{\mathbf{x}}_{2}$ and the axial vectors of $\Delta \mathbf{Q}_{1}$ and $\Delta \mathbf{Q}_{2}$. That is,

$$
\left[\begin{array}{l}
\Delta \mathrm{F}_{1} \\
\Delta \mathrm{F}_{2}
\end{array}\right]=-{ }_{1} \mathrm{~K}_{c}\left[\begin{array}{c}
\Delta \mathrm{x}^{1} \\
\Delta \mathrm{x}^{2}
\end{array}\right]+O\left(\epsilon^{2}\right),
$$

where $\Delta \mathrm{F}_{K}$ and $\Delta \mathrm{x}^{K}$ are given by

$$
\Delta \mathbf{F}_{K}=\left[\begin{array}{c}
\left(\mathbf{F}_{K}^{\prime}-\mathbf{F}_{K}\right) \cdot \mathbf{E}_{1} \\
\left(\mathbf{F}_{K}^{\prime}-\mathbf{F}_{K}\right) \cdot \mathbf{E}_{2} \\
\left(\mathbf{F}_{K}^{\prime}-\mathbf{F}_{K}\right) \cdot \mathbf{E}_{3} \\
\left(\mathbf{M}_{K, O}^{\prime}-\mathbf{M}_{K, O}\right) \cdot \mathbf{E}_{1} \\
\left(\mathbf{M}_{K, O}^{\prime}-\mathbf{M}_{K, O}\right) \cdot \mathbf{E}_{2} \\
\left(\mathbf{M}_{K, O}^{\prime}-\mathbf{M}_{K, O}\right) \cdot \mathbf{E}_{3}
\end{array}\right], \quad \Delta \mathbf{x}^{K}=\left[\begin{array}{c}
\Delta \overline{\mathbf{x}}^{K} \cdot \mathbf{E}_{1} \\
\Delta \overline{\mathbf{x}}^{K} \cdot \mathbf{E}_{2} \\
\Delta \overline{\mathbf{x}}^{K} \cdot \mathbf{E}_{3} \\
\Delta \boldsymbol{\theta}_{K} \cdot \mathbf{E}_{1} \\
\Delta \boldsymbol{\theta}_{K} \cdot \mathbf{E}_{2} \\
\Delta \boldsymbol{\theta}_{K} \cdot \mathbf{E}_{3}
\end{array}\right]
$$

Related remarks pertain to the $U_{4}$ case. 
8.5 The case $U=\hat{U}_{5}(\mathbf{R}, \mathbf{y})$

When the potential energy acting on the pair of rigid bodies is independent of the surroundings (cf. Fig. 6(c)), the potential energy simplifies dramatically to $U=U_{5}(\mathbf{y}, \mathbf{R})$. Following the same line of argument that led to (90), we conclude that

$$
\mathbf{F}_{2}=-\mathbf{F}_{1}=-\sum_{k=1}^{3} \frac{\partial U_{5}}{\partial Y_{k}} \mathbf{E}_{k}, \quad \mathbf{M}_{2}=-\mathbf{M}_{1}=-\sum_{k=1}^{3} \frac{\partial U_{5}}{\partial \beta^{k}} \mathbf{g}^{\mathrm{rel}, k}
$$

The Cartesian stiffness matrix for this case can be expressed as a $6 \times 6$ matrix which relates the components of $\mathbf{F}_{2}$ and $\mathbf{M}_{2}$ to the increments in $\Delta \boldsymbol{\theta}$ and $\mathbf{y} \cdot \mathbf{E}_{i}$. The vector $\Delta \boldsymbol{\theta}$ is the axial vector of $\Delta \mathbf{R}$. The resulting stiffness matrix will be similar in form to the matrix ${ }_{2} \mathrm{~K}_{c}$ discussed in Sect. 4.2.

\subsection{Incorporating constraints}

In certain situations, the two rigid bodies may be connected by joints (e.g., as in Fig. 6(b)). This situation can be accommodated by appropriately selecting the point $A$ on each body to coincide with the joint:

$$
\mathbf{x}_{A}=\mathbf{x}_{A}^{1}=\mathbf{x}_{A}^{2},
$$

and, if needed, constraining the angles $\beta^{1}, \beta^{2}$, and possibly $\beta^{3}$. To establish the stiffness matrix for this case, we consider $U=\hat{U}_{6}\left(\mathbf{Q}_{1}, \mathbf{R}, \mathbf{x}_{A}\right)$. Simplifying (86) with the help of (91), we seek solutions of

$$
-\dot{U}_{6}=\mathbf{F}_{1} \cdot \dot{\mathbf{x}}_{A}^{1}+\mathbf{F}_{2} \cdot \dot{\mathbf{x}}_{A}^{2}+\mathbf{M}_{1, A} \cdot \boldsymbol{\omega}_{1}+\mathbf{M}_{2, A} \cdot \boldsymbol{\omega}_{2},
$$

subject to the constraints

$$
\dot{\mathbf{x}}_{A}^{1}=\dot{\mathbf{x}}_{A}^{2} .
$$

Using a standard procedure, the solution is

$$
\begin{aligned}
\mathbf{F}_{1}+\mathbf{F}_{2} & =-\sum_{k=1}^{3} \frac{\partial U_{6}}{\partial X_{A, k}} \mathbf{E}_{k}, \\
\mathbf{M}_{1, A} & =-\sum_{k=1}^{3} \frac{\partial U_{6}}{\partial v_{1}^{k}} \mathbf{g}^{1, k}+\sum_{k=1}^{3} \frac{\partial U_{6}}{\partial \beta^{k}} \mathbf{g}^{\mathrm{rel}, k}, \\
\mathbf{M}_{2, A} & =-\sum_{k=1}^{3} \frac{\partial U_{6}}{\partial \beta^{k}} \mathbf{g}^{\mathrm{rel}, k} .
\end{aligned}
$$

The Cartesian stiffness matrix in this case will be a $9 \times 9$ matrix which will relate the Cartesian components $\left(\mathbf{F}_{1}+\mathbf{F}_{2}\right) \cdot \mathbf{E}_{k}, \mathbf{M}_{1, A} \cdot \mathbf{E}_{k}$, and $\mathbf{M}_{2, A} \cdot \mathbf{E}_{k}$ to the Cartesian components of $\mathbf{x}_{A}$, and the axial vectors of $\Delta \mathbf{Q}_{1}$ and $\Delta \mathbf{R}$.

Because of the joint at A, the identity (101) does not yield the individual conservative forces acting on the bodies rather it yields the resultant conservative force acting on the system of two rigid bodies. To elaborate, reaction forces $\mathbf{N}_{1}$ and $\mathbf{N}_{2}$ will act on the respective bodies at the joint $A$. These forces will be equal and opposite: $\mathbf{N}_{1}=-\mathbf{N}_{2}$. The resultant 
forces on the first body due to the joint at $A$ and the conservative forces is $\mathbf{N}_{1}+\mathbf{F}_{1}$, while the corresponding resultant force on the second body is $\mathbf{N}_{2}+\mathbf{F}_{2}$. As $\mathbf{N}_{1}$ and $\mathbf{N}_{2}$ are nonconservative, they are not prescribed by (101) and so we can only use (101) to determine $\mathbf{F}_{1}+\mathbf{F}_{2}$.

\section{A planar multibody system}

In the interest of brevity, we focus our attention on a multibody system undergoing planar motions in the $\mathbf{E}_{1}-\mathbf{E}_{2}$ plane as shown in Fig. 7. For the planar case, each body only has a single angle of rotation, $\psi_{J}$. As in Sect. 6, the axis of rotation for each of the $N$ bodies is simply $\mathbf{E}_{3}$.

Before proceeding, we first recall the following notation. Associated with each of the $K$ rigid bodies is the set of basis vectors given by $\mathbf{e}_{i}^{J}=\mathbf{Q}_{J} \mathbf{E}_{i}$, where $\mathbf{Q}_{J}$ is the rotation tensor associated with the $J$ th rigid body. The position vector of the center of mass of each rigid body is then be written as

$$
\overline{\mathbf{x}}^{J}=\sum_{i=1}^{3} x_{i}^{J} \mathbf{e}_{i}^{J}=\sum_{i=1}^{3} X_{i}^{J} \mathbf{E}_{i} \quad(J=1, \ldots, N) .
$$

We will follow Sect. 8 and use lower case letters to express the components of $\overline{\mathbf{x}}^{J}$ with respect to the basis vectors fixed to its body.

Once again, we assume that the potential energy can be written as functions of the coordinates and rotation angles. However, we will focus only on the potential energy functions that can be written as a function of the absolute as opposed to the relative position vectors since the ensuing representation are more amenable to deriving the associated Cartesian stiffness matrix associated with each rigid body. Thus, we have

$$
\begin{aligned}
U & =U_{1}\left(\psi_{1}, x_{1}^{1}, x_{2}^{1}, \ldots, \psi_{N}, x_{1}^{N}, x_{2}^{N}\right) \\
& =U_{2}\left(\psi_{1}, X_{1}^{1}, X_{2}^{1}, \ldots, \psi_{N}, X_{1}^{N}, X_{2}^{N}\right)
\end{aligned}
$$

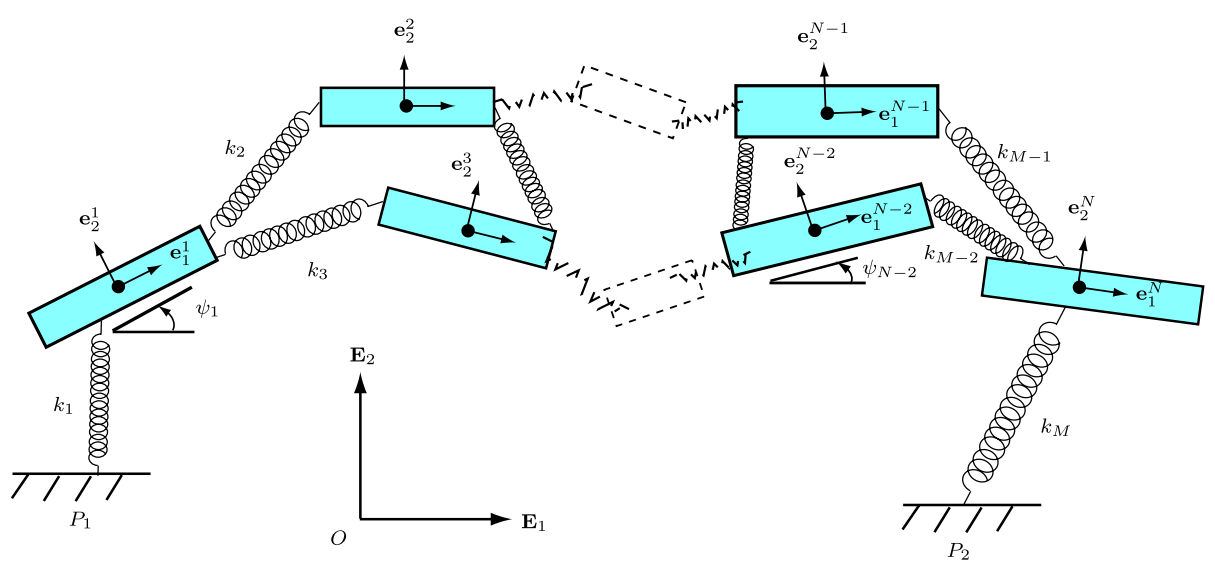

Fig. 7 Schematic of a system of $N$ rigid bodies undergoing planar motion. The system is attached to two fixed points $P_{1}$ and $P_{2}$ and to each other by a system of springs 


$$
\begin{aligned}
& =U_{4}\left(\psi_{1}, X_{A_{1}}^{1}, X_{A_{2}}^{1}, \ldots, \psi_{N}, X_{A_{1}}^{N}, X_{A_{2}}^{N}\right) \\
& =U_{6}\left(\psi_{1}, X_{A_{1}}^{1}, X_{A_{2}}^{1}, \psi_{2}-\psi_{1}, \ldots, \psi_{N}-\psi_{N-1}, X_{A_{1}}^{N}, X_{A_{2}}^{N}\right) .
\end{aligned}
$$

Associated with these potential energy functions are the respective Cartesian stiffness matrices ${ }_{1} \mathrm{~K}_{c},{ }_{2} \mathrm{~K}_{c},{ }_{4} \mathrm{~K}_{c}$, and ${ }_{6} \mathrm{~K}_{c}$. Notice that the representation $U=\hat{U}_{5}$ cannot be used in this example since the bodies are connected to the ground by springs.

The potential energy function for the system can be obtained by adding the potential energies of each of the $M$ springs connecting the system:

$$
V=\sum_{p=1}^{M} \frac{k_{p}}{2}\left(l_{p}-l_{0 p}\right)^{2},
$$

where $l_{01}, \ldots, l_{0 M}$ and $l_{1}, \ldots, l_{M}$ are the unstretched and stretched lengths of the springs respectively, written as functions of the coordinates of the rigid bodies, $\psi_{1}, x_{1}^{1}, x_{2}^{1}, \ldots, \psi_{N}, x_{1}^{N}$, and $x_{2}^{N}$. That is, $V=U_{1}$. Paralleling the developments of Sects. 4.1 and 8.4 and performing Taylor series expansions for $\mathbf{F}_{J}$ and $\mathbf{M}_{J, O}$ about their equilibrium configurations, we can write

$$
\left[\begin{array}{c}
\Delta \mathrm{F}_{1} \\
\Delta \mathrm{F}_{2} \\
\vdots \\
\Delta \mathrm{F}_{N-1} \\
\Delta \mathrm{F}_{N}
\end{array}\right]=-{ }_{1} \mathrm{~K}_{c}\left[\begin{array}{c}
\Delta \mathrm{x}^{1} \\
\Delta \mathrm{x}^{2} \\
\vdots \\
\Delta \mathrm{x}^{N-1} \\
\Delta \mathrm{x}^{N}
\end{array}\right]+O\left(\epsilon^{2}\right)
$$

where

$$
\begin{aligned}
\Delta \mathrm{F}_{J}= & {\left[\begin{array}{c}
\left(\mathbf{F}_{J}^{\prime}-\mathbf{F}_{J}\right) \cdot \mathbf{E}_{1} \\
\left(\mathbf{F}_{J}^{\prime}-\mathbf{F}_{J}\right) \cdot \mathbf{E}_{2} \\
\left(\mathbf{M}_{J, O}^{\prime}-\mathbf{M}_{J, O}\right) \cdot \mathbf{E}_{3}
\end{array}\right], \quad \Delta \mathbf{x}^{J}=\left[\begin{array}{c}
\Delta \overline{\mathbf{x}}^{J} \cdot \mathbf{E}_{1} \\
\Delta \overline{\mathbf{x}}^{J} \cdot \mathbf{E}_{2} \\
\Delta \psi_{J}
\end{array}\right] \cdot } \\
{ }_{1} \mathrm{~K}_{c}= & {\left[\begin{array}{ccc}
\mathrm{Q}_{1} & 0 & 0 \\
0 & \ddots & 0 \\
0 & 0 & \mathrm{Q}_{N}
\end{array}\right]\left[\begin{array}{ccc}
\mathrm{H}_{1,1} & \ldots & \mathrm{H}_{1, N} \\
\vdots & \ddots & \vdots \\
\mathrm{H}_{N, 1} & \ldots & \mathrm{H}_{N, N}
\end{array}\right]\left[\begin{array}{ccc}
\mathrm{Q}_{1} & 0 & 0 \\
0 & \ddots & 0 \\
0 & 0 & \mathrm{Q}_{N}
\end{array}\right]+\left[\begin{array}{ccc}
\mathrm{W}_{1} & 0 & 0 \\
0 & \ddots & 0 \\
0 & 0 & \mathrm{~W}_{N}
\end{array}\right] } \\
& +\left[\begin{array}{ccc}
\mathrm{Q}_{1} & 0 & 0 \\
0 & \ddots & 0 \\
0 & 0 & \mathrm{Q}_{N}
\end{array}\right]^{T}\left[\begin{array}{ccc}
\mathrm{H}_{1,1} & \ldots & \mathrm{H}_{1, N} \\
\vdots & \ddots & \vdots \\
\mathrm{H}_{N, 1} & \ldots & \mathrm{H}_{N, N}
\end{array}\right]\left[\begin{array}{ccc}
\mathrm{S}_{1} & 0 & 0 \\
0 & \ddots & 0 \\
0 & 0 & \mathrm{~S}_{N}
\end{array}\right]\left[\begin{array}{ccc}
\mathrm{Q}_{1} & 0 & 0 \\
0 & \ddots & 0 \\
0 & 0 & \mathrm{Q}_{N}
\end{array}\right]
\end{aligned}
$$

with

$$
\mathrm{H}_{I, J}=\left[\begin{array}{ccc}
\frac{\partial^{2} U_{1}}{\partial x_{1}^{I} \partial x_{1}^{J}} & \frac{\partial^{2} U_{1}}{\partial x_{1}^{I} \partial x_{2}^{J}} & \frac{\partial^{2} U_{1}}{\partial x_{1}^{I} \partial \psi_{J}} \\
\frac{\partial^{2} U_{1}}{\partial x_{2}^{I} \partial x_{1}^{J}} & \frac{\partial^{2} U_{1}}{\partial x_{2}^{I} \partial x_{2}^{J}} & \frac{\partial^{2} U_{1}}{\partial x_{2}^{I} \partial \psi_{J}} \\
\frac{\partial^{2} U_{1}}{\partial \psi_{I} \partial x_{1}^{J}} & \frac{\partial^{2} U_{1}}{\partial \psi_{I} \partial x_{2}^{J}} & \frac{\partial^{2} U_{1}}{\partial \psi_{I} \partial \psi_{J}}
\end{array}\right] \quad(I, J=1, \ldots, N),
$$


$\mathrm{Q}_{K}$ is the rotation matrix associated with the $K$ th rigid body,

$$
\mathrm{Q}_{K}=\left[\begin{array}{ccc}
\cos \left(\psi_{K}\right) & \sin \left(\psi_{K}\right) & 0 \\
-\sin \left(\psi_{K}\right) & \cos \left(\psi_{K}\right) & 0 \\
0 & 0 & 1
\end{array}\right]
$$

$\mathrm{S}_{K}$ is the skew-symmetric matrix with components given by

$$
\mathrm{S}_{K, i j}=\left(\mathbf{e}_{j}^{K} \times \mathbf{e}_{i}^{K}\right) \cdot \overline{\mathbf{x}}^{K},
$$

and, as in Sect. 6, the asymmetric components of ${ }_{1} \mathrm{~K}_{c}$ have been written in terms of force components:

$$
\mathrm{W}_{K}=\left[\begin{array}{ccc}
0 & 0 & \mathbf{F}_{K} \cdot \mathbf{E}_{2} \\
0 & 0 & -\mathbf{F}_{K} \cdot \mathbf{E}_{1} \\
0 & 0 & 0
\end{array}\right] .
$$

If the force $\mathbf{F}$ and moment $\mathbf{M}$ are used instead, then we need to repeat the calculation with the function $U_{2}$. In this case, we obtain a symmetric Cartesian stiffness matrix:

$$
\left[\begin{array}{c}
\Delta \mathrm{F}_{1} \\
\Delta \mathrm{F}_{2} \\
\vdots \\
\Delta \mathrm{F}_{N-1} \\
\Delta \mathrm{F}_{N}
\end{array}\right]=-\left[\begin{array}{ccccc}
\tilde{\mathrm{H}}_{1,1} & \tilde{\mathrm{H}}_{1,2} & \cdots & \tilde{\mathrm{H}}_{1, N-1} & \tilde{\mathrm{H}}_{1, N} \\
\tilde{\mathrm{H}}_{2,1} & \tilde{\mathrm{H}}_{2,2} & \cdots & \tilde{\mathrm{H}}_{2, N-1} & \tilde{\mathrm{H}}_{2, N} \\
\vdots & & \ddots & & \vdots \\
\tilde{\mathrm{H}}_{N-1,1} & \tilde{\mathrm{H}}_{N-1,2} & \cdots & \tilde{\mathrm{H}}_{N-1, N-1} & \tilde{\mathrm{H}}_{N-1, N} \\
\tilde{\mathrm{H}}_{N, 1} & \tilde{\mathrm{H}}_{N, 2} & \cdots & \tilde{\mathrm{H}}_{N, N-1} & \tilde{\mathrm{H}}_{N, N}
\end{array}\right]\left[\begin{array}{c}
\Delta \mathrm{x}^{1} \\
\Delta \mathrm{x}^{2} \\
\vdots \\
\Delta \mathbf{x}^{N-1} \\
\Delta \mathbf{x}^{N}
\end{array}\right]+O\left(\epsilon^{2}\right)
$$

The $3 K \times 3 K$ matrix in this equation is the (symmetric) Cartesian stiffness matrix ${ }_{2} \mathrm{~K}_{c}$ with components, $\tilde{\mathrm{H}}_{I J}$ given by

$$
\tilde{\mathrm{H}}_{I, J}=\left[\begin{array}{ccc}
\frac{\partial^{2} U_{1}}{\partial X_{1}^{I} \partial X_{1}^{J}} & \frac{\partial^{2} U_{1}}{\partial X_{1}^{I} \partial X_{2}^{J}} & \frac{\partial^{2} U_{1}}{\partial X_{1}^{I} \partial \psi_{J}} \\
\frac{\partial^{2} U_{1}}{\partial X_{2}^{I} \partial X_{1}^{J}} & \frac{\partial^{2} U_{1}}{\partial X_{2}^{I} \partial X_{2}^{J}} & \frac{\partial^{2} U_{1}}{\partial X_{2}^{I} \partial \psi_{J}} \\
\frac{\partial^{2} U_{1}}{\partial \psi_{I} \partial X_{1}^{J}} & \frac{\partial^{2} U_{1}}{\partial \psi_{I} \partial X_{2}^{J}} & \frac{\partial^{2} U_{1}}{\partial \psi_{I} \partial \psi_{J}}
\end{array}\right] .
$$

\section{Closing remarks}

In this paper, it is shown how various representations for Cartesian stiffness matrices $\mathrm{K}_{c}$ are obtained for a wide range of pairs of resultant forces and moments. The selection of the pair of forces and moments is not arbitrary: rather it is related by a work argument to the functional representation of the potential energy function (see (10) and (14)):

$$
\begin{aligned}
\left(\mathbf{F}, \mathbf{M}_{O}\right) & \rightarrow U_{1}\left(v^{1}, v^{2}, v^{3}, x_{1}, x_{2}, x_{3}\right), \\
(\mathbf{F}, \mathbf{M}) & \rightarrow U_{2}\left(v^{1}, v^{2}, v^{3}, X_{1}, X_{2}, X_{3}\right), \\
\left(\mathbf{F}_{A}, \mathbf{M}_{O}\right) & \rightarrow U_{3}\left(v^{1}, v^{2}, v^{3}, x_{A_{1}}, x_{A_{2}}, x_{A_{3}}\right), \\
\left(\mathbf{F}_{A}, \mathbf{M}_{A}\right) & \rightarrow U_{4}\left(v^{1}, v^{2}, v^{3}, X_{A_{1}}, X_{A_{2}}, X_{A_{3}}\right) .
\end{aligned}
$$


We also remark that the use of the dual Euler basis to calculate $\mathrm{K}_{c}$ was an essential component of the formulation. Should a quaternion or Euler-Rodrigues symmetric parameter representation of the rotation be used, then it is possible to extend the formulation presented in this paper to that case. The formulation would use representations for the conservative moments that can be found in [6].

Acknowledgements The work of the authors was partially supported by the National Science Foundation under Grant No. CMMI 0726675. Melodie Metzger was also supported by a Graduate Fellowship from the National Science Foundation. The authors express their thanks to Tim Gasperak for his assistance with Fig. 4, and the reviewers for their constructive criticisms.

Open Access This article is distributed under the terms of the Creative Commons Attribution Noncommercial License which permits any noncommercial use, distribution, and reproduction in any medium, provided the original author(s) and source are credited.

\section{Appendix A: Derivatives of the corotational basis vectors and the Euler basis vectors}

The Euler basis vectors are parallel to the three axes of rotation which are used to define the Euler angles. Thus, if $v^{1}, v^{2}$, and $v^{3}$ are the three Euler angles, then the rotation tensor $\mathbf{Q}=\mathbf{Q}\left(v^{1}, v^{2}, v^{3}\right)$. As $\mathbf{Q}$ is a rotation tensor: $\mathbf{Q} \mathbf{Q}^{T}=\mathbf{I}$, where $\mathbf{I}$ is the identity tensor and the superscript $T$ denotes the transpose. Consequently, $\mathbf{Q} \mathbf{Q}^{T}$ is a skew-symmetric tensor. Every skew-symmetric tensor $\mathbf{A}$ has a unique axial vector $\mathbf{a}$ where $\mathbf{A b}=\mathbf{a} \times \mathbf{b}: \mathbf{a}=\operatorname{ax}(\mathbf{A})$. The axial vector of $\dot{\mathbf{Q}} \mathbf{Q}^{T}$ is the angular velocity vector $\boldsymbol{\omega}$ and this vector has the representations

$$
\begin{aligned}
\boldsymbol{\omega}=\operatorname{ax}\left(\dot{\mathbf{Q}} \mathbf{Q}^{T}\right) & =\sum_{k=1}^{3} \dot{v}^{k} \operatorname{ax}\left(\frac{\partial \mathbf{Q}}{\partial v^{k}} \mathbf{Q}^{T}\right) \\
& =\sum_{k=1}^{3} \dot{v}^{k} \mathbf{g}_{k} .
\end{aligned}
$$

From the representations (117), it should be clear that we can identify $\mathbf{g}_{k}$ as the axial vector of the skew-symmetric tensor $\boldsymbol{\Omega}_{k}$ :

$$
\mathbf{g}_{k}=\operatorname{ax}\left(\boldsymbol{\Omega}_{k}\right), \quad \boldsymbol{\Omega}_{k}=-\boldsymbol{\Omega}_{k}^{T}=\frac{\partial \mathbf{Q}}{\partial v^{k}} \mathbf{Q}^{T} .
$$

The partial derivatives of the corotational vector $\mathbf{e}_{i}=\mathbf{Q E}_{i}$ with respect to the Euler angles play a key role in the development of the stiffness matrix. Computing $\frac{\partial \mathbf{e}_{i}}{\partial \nu^{k}}$ and appealing to (118), it is easy to show that

$$
\frac{\partial \mathbf{e}_{i}}{\partial v^{k}}=\mathbf{g}_{k} \times \mathbf{e}_{i} .
$$

This identity is used to establish (48) in Sect. 5. This expression for the partial derivative of $\mathbf{e}_{i}$ complements the more traditional representation

$$
\frac{\partial \mathbf{e}_{i}}{\partial \nu^{k}}=\sum_{r=1}^{3} \frac{\partial Q_{i r}}{\partial \nu^{k}} \mathbf{E}_{r}
$$

which is obtained by differentiating $\mathbf{e}_{i}=\sum_{r=1}^{3} Q_{i r} \mathbf{E}_{r}$.

The second set of derivatives which play a key role in the stiffness matrix are $\frac{\partial \mathbf{g}_{k}}{\partial \nu^{i}}$. Here, we derive an identity which is used to establish (56) and (58) in Sect. 5. First, we compute 
the derivative of $\mathbf{g}_{k}$ and find that

$$
\begin{aligned}
\frac{\partial \mathbf{g}_{k}}{\partial v^{j}} & =\operatorname{ax}\left(\frac{\partial^{2} \mathbf{Q}}{\partial v^{j} v^{k}} \mathbf{Q}^{T}\right)+\operatorname{ax}\left(\frac{\partial \mathbf{Q}}{\partial v^{k}} \frac{\partial \mathbf{Q}^{T}}{\partial v^{j}}\right) \\
& =\operatorname{ax}\left(\frac{\partial^{2} \mathbf{Q}}{\partial v^{j} v^{k}} \mathbf{Q}^{T}\right)+\operatorname{ax}\left(\frac{\partial \mathbf{Q}}{\partial v^{k}} \mathbf{Q}^{T} \mathbf{Q} \frac{\partial \mathbf{Q}^{T}}{\partial v^{j}}\right) \\
& =\operatorname{ax}\left(\frac{\partial^{2} \mathbf{Q}}{\partial v^{j} v^{k}} \mathbf{Q}^{T}\right)+\operatorname{ax}\left(\boldsymbol{\Omega}_{k} \boldsymbol{\Omega}_{j}^{T}\right) .
\end{aligned}
$$

Invoking the identities $\frac{\partial^{2} \mathbf{Q}}{\partial v^{j} v^{k}}=\frac{\partial^{2} \mathbf{Q}}{\partial \nu^{k} v^{j}}$ and $\boldsymbol{\Omega}_{j}^{T}=-\boldsymbol{\Omega}_{j}$, it follows that

$$
\frac{\partial \mathbf{g}_{k}}{\partial v^{j}}-\frac{\partial \mathbf{g}_{j}}{\partial v^{k}}=\operatorname{ax}\left(\boldsymbol{\Omega}_{j} \boldsymbol{\Omega}_{k}-\boldsymbol{\Omega}_{k} \boldsymbol{\Omega}_{j}\right) .
$$

From (118), we note that the axial vectors of $\boldsymbol{\Omega}_{j}$ and $\boldsymbol{\Omega}_{k}$ are, respectively, $\mathbf{g}_{j}$ and $\mathbf{g}_{k}$. With the help of a well-known identity ${ }^{3}$ for the axial vector of a product of the form $\boldsymbol{\Omega}_{j} \boldsymbol{\Omega}_{k}-$ $\boldsymbol{\Omega}_{k} \boldsymbol{\Omega}_{j}$, we conclude that

$$
\frac{\partial \mathbf{g}_{k}}{\partial v^{j}}-\frac{\partial \mathbf{g}_{j}}{\partial v^{k}}=\mathbf{g}_{j} \times \mathbf{g}_{k}
$$

\section{Appendix B: The 3-2-1 set of Euler angles}

For the 3-2-1 set of Euler angles, we denote $v^{1}=\psi, v^{2}=\theta$, and $v^{3}=\phi$ (see Fig. 8). This set of Euler angles is commonly used in biomechanics and vehicle dynamics, and is discussed in numerous textbooks. Here, we recall some results for this set from [13, 14].

Fig. 8 Schematic of the 3-2-1 set of Euler angles and the individual rotations these angles represent. In this figure, the three Euler angles are denoted by $\psi=v^{1}, \theta=v^{2}$ and $\phi=v^{3}$, respectively, and the rotation tensor $\mathbf{Q}$ that they parameterize transforms $\mathbf{E}_{i}$ to $\mathbf{e}_{i}$

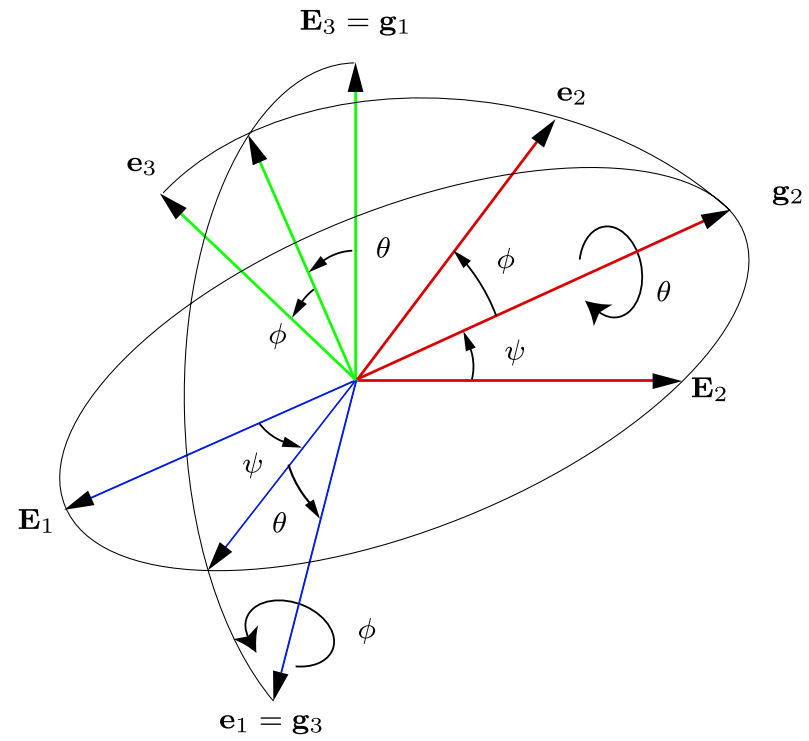

${ }^{3}$ See Example A.7 in [11] or Eq. (A.3) $)_{1}$ in [12]. 
Recalling that $Q_{k i}=\mathbf{e}_{k} \cdot \mathbf{E}_{i}$, one can compute the components of the matrix Q:

$$
\begin{aligned}
{\left[\begin{array}{lll}
Q_{11} & Q_{12} & Q_{13} \\
Q_{21} & Q_{22} & Q_{23} \\
Q_{31} & Q_{32} & Q_{33}
\end{array}\right]=} & {\left[\begin{array}{ccc}
1 & 0 & 0 \\
0 & \cos (\phi) & \sin (\phi) \\
0 & -\sin (\phi) & \cos (\phi)
\end{array}\right]\left[\begin{array}{ccc}
\cos (\theta) & 0 & -\sin (\theta) \\
0 & 1 & 0 \\
\sin (\theta) & 0 & \cos (\theta)
\end{array}\right] } \\
& \times\left[\begin{array}{ccc}
\cos (\psi) & \sin (\psi) & 0 \\
-\sin (\psi) & \cos (\psi) & 0 \\
0 & 0 & 1
\end{array}\right] .
\end{aligned}
$$

The Euler basis vectors $\left\{\mathbf{g}_{1}, \mathbf{g}_{2}, \mathbf{g}_{3}\right\}$ have the representations

$$
\left[\begin{array}{l}
\mathbf{g}_{1} \\
\mathbf{g}_{2} \\
\mathbf{g}_{3}
\end{array}\right]=\left[\begin{array}{ccc}
0 & 0 & 1 \\
-\sin (\psi) & \cos (\psi) & 0 \\
\cos (\theta) \cos (\psi) & \cos (\theta) \sin (\psi) & -\sin (\theta)
\end{array}\right]\left[\begin{array}{l}
\mathbf{E}_{1} \\
\mathbf{E}_{2} \\
\mathbf{E}_{3}
\end{array}\right] .
$$

The components $G_{k}^{i}=\mathbf{g}^{i} \cdot \mathbf{E}_{k}$ of the matrix $\mathrm{G}$ can be inferred from the following representations for the dual Euler basis vectors:

$$
\left[\begin{array}{l}
\mathbf{g}^{1} \\
\mathbf{g}^{2} \\
\mathbf{g}^{3}
\end{array}\right]=\left[\begin{array}{ccc}
\cos (\psi) \tan (\theta) & \sin (\psi) \tan (\theta) & 1 \\
-\sin (\psi) & \cos (\psi) & 0 \\
\cos (\psi) \sec (\theta) & \sin (\psi) \sec (\theta) & 0
\end{array}\right]\left[\begin{array}{l}
\mathbf{E}_{1} \\
\mathbf{E}_{2} \\
\mathbf{E}_{3}
\end{array}\right]
$$

The matrix featuring on the right-hand side of (125) is $\mathrm{G}^{-T}$. Notice that $\mathbf{g}^{i} \cdot \mathbf{g}_{k}=\delta_{k}^{i}$ where $\delta_{k}^{i}$ is the Kronecker delta.

It is straightforward to show from (126) that

$$
\begin{aligned}
& \frac{\partial \mathbf{g}^{1}}{\partial \psi}=\tan (\theta) \mathbf{g}^{2}, \quad \frac{\partial \mathbf{g}^{2}}{\partial \psi}=-\cos (\theta) \mathbf{g}^{3}, \quad \frac{\partial \mathbf{g}^{3}}{\partial \psi}=\sec (\theta) \mathbf{g}^{2}, \\
& \frac{\partial \mathbf{g}^{1}}{\partial \theta}=\sec (\theta) \mathbf{g}^{3}, \quad \frac{\partial \mathbf{g}^{2}}{\partial \theta}=\mathbf{0}, \quad \frac{\partial \mathbf{g}^{3}}{\partial \theta}=\tan (\theta) \mathbf{g}^{3}, \quad \frac{\partial \mathbf{g}^{k}}{\partial \phi}=\mathbf{0} .
\end{aligned}
$$

From these equations, we can compute the connection coefficients:

$$
\gamma_{j k}^{i}=-\frac{\partial \mathbf{g}^{i}}{\partial v^{k}} \cdot \mathbf{g}_{j}
$$

Most of these 27 coefficients are zero, and so we only record the non-trivial ones:

$$
\begin{gathered}
\gamma_{21}^{1}=-\tan (\theta), \quad \gamma_{32}^{1}=-\sec (\theta), \quad \gamma_{31}^{2}=\cos (\theta), \\
\gamma_{21}^{3}=-\sec (\theta), \quad \gamma_{32}^{3}=-\tan (\theta) .
\end{gathered}
$$

It should be noticed that the coefficients $\gamma_{k j}^{i}$ do not possess the symmetry $\gamma_{k j}^{i}=\gamma_{j k}^{i}$ that is found in the Christoffel symbols $\Gamma_{k j}^{i}$ of the second kind.

\section{References}

1. Andriacchi, T.P., Mikosz, R.P., Hampton, S.J., Galante, J.O.: Model studies of the stiffness characteristics of the human knee joint. J. Biomech. 16(1), 23-29 (1983). doi:10.1016/0021-9290(83)90043-X 
2. Bishop, R.L., Goldberg, S.I.: Tensor Analysis on Manifolds. Dover, New York (1980)

3. Chen, S.F., Kao, I.: Conservative congruence transformation for joint and Cartesian stiffness matrices of robotic hands and fingers. Int. J. Robotics Res. 19(9), 835-847 (2000). doi:10.1177/ 02783640022067201

4. Choquet-Bruhat, Y., DeWitt-Morette, C., Dillard Bleick, M.: Analysis, Manifolds, and Physics, revised edn. North-Holland Physics Publishing, Amsterdam (1982)

5. Ciblak, N., Lipkin, H.: Asymmetric Cartesian stiffness for the modelling of compliant robotic systems. In: Pennock, G.R., Angeles, J., Fichter, E.F., Freeman, R.A., Lipkin, H., Thompson, B.S., Wiederrich, J., Wiens, G.L. (eds.) Robotics: Kinematics, Dynamics and Controls, Presented at The 1994 ASME Design Technical Conferences-23rd Biennial Mechanisms Conference, Minneapolis, Minnesota, September 11-14, 1994, vol. DE-72, pp. 197-204. ASME, New York (1994)

6. Faruk Senan, N.A., O'Reilly, O.M.: On the use of quaternions and Euler-Rodrigues symmetric parameters with moments and moment potentials. Int. J. Eng. Sci. 47(4), 595-609 (2009). doi:10.1016/ j.ijengsci.2008.12.008

7. Gardner-Morse, M.G., Stokes, I.A.F.: Structural behavior of the human lumbar spinal motion segments. J. Biomech. 37(2), 205-212 (2004). doi:10.1016/j.jbiomech.2003.10.003

8. Griffis, M., Duffy, J.: Global stiffness modeling of a class of simple compliant couplings. Mech. Mach. Theory 28(2), 207-224 (1993). doi:10.1016/0094-114X(93)90088-D

9. Howard, S., Žefran, M., Kumar, V.: On the $6 \times 6$ Cartesian stiffness matrix for three-dimensional motions. Mech. Mach. Theory 33(4), 389-408 (1998). doi:10.1016/S0094-114X(97)00040-2

10. Kövecses, J., Angeles, J.: The stiffness matrix in elastically articulated rigid-body systems. Multibody Syst. Dyn. 18(2), 169-184 (2007). doi:10.1007/s11044-007-9082-2

11. Murray, R.N., Li, Z.X., Sastry, S.S.: A Mathematical Introduction to Robotic Manipulation. CRC Press, Boca Raton (1994)

12. Nordenholz, T.R., O'Reilly, O.M.: A class of motions of elastic, symmetric Cosserat points: existence, bifurcation, and stability. Int. J. Non-Linear Mech. 36(2), 353-374 (2001). doi:10.1016/ S0020-7462(00)00021-4

13. O'Reilly, O.M.: The dual Euler basis: Constraints, potentials, and Lagrange's equations in rigid body dynamics. ASME J. Appl. Mech. 74(2), 256-258 (2007). doi:10.1115/1.2190231

14. O'Reilly, O.M.: Intermediate Engineering Dynamics: A Unified Approach to Newton-Euler and Lagrangian Mechanics. Cambridge University Press, New York (2008). http://www.cambridge.org/us/ catalogue/catalogue.asp?isbn=9780521874830

15. O'Reilly, O.M., Srinivasa, A.R.: On potential energies and constraints in the dynamics of rigid bodies and particles. Math. Probl. Eng. 8(3), 169-180 (2002). doi:10.1080/10241230215286

16. O'Reilly, O.M., Metzger, M.F., Buckley, J.M., Moody, D.A., Lotz, J.C.: On the stiffness matrix of the intervertebral joint: application to total disk replacement. J. Biomech. Eng. 131(8), 63-87 (2009). doi:10.1115/1.3148195

17. Panjabi, M.M., Brand, R.A. Jr., White, A.A. III: Three-dimensional flexibility and stiffness properties of the human thoracic spine. J. Biomech. 9(4), 185-192 (1976). doi:10.1016/0021-9290(76)90003-8

18. Pigoski, T., Griffis, M., Duffy, J.: Stiffness mappings employing different frames of reference. Mech. Mach. Theory 33(6), 825-838 (1998). doi:10.1016/S0094-114X(97)00083-9

19. Quennouelle, C., Gosselin, C.M.: Stiffness matrix of compliant parallel mechanisms. In: Lenarčič, J., Wenger, P. (eds.) Advances in Robot Kinematics: Analysis and Design, pp. 331-341. Springer, Dordrecht (2008). doi:10.1007/978-1-4020-8600-7_35

20. Shuster, M.D.: A survey of attitude representations. J. Astronaut. Sci. 41(4), 439-517 (1993)

21. Žefran, M., Kumar, V.: A geometrical approach to the study of the Cartesian stiffness matrix. ASME J. Mech. Des. 124(1), 30-38 (2002). doi:10.1115/1.1423638 